\title{
EVALUATION OF THE INNOVATIVE VALUE PROPOSITION FOR THE RAIL FREIGHT TRANSPORT: AN INTEGRATED DEMATEL-ANP APPROACH
}

\author{
Miloš MILENKOVIĆ ${ }^{1,2^{*}}$, Susana VAL ${ }^{3}$, Dejan LUTOVAC ${ }^{4}$, \\ Nebojša BOJOVIĆ ${ }^{5}$ Nikola KNEŽEVIĆ 6 \\ 1,4,5, ${ }^{6}$ Faculty of Transport and Traffic Engineering, University of Belgrade, Belgrade, Serbia \\ ${ }^{2,3}$ Zaragoza Logistics Center, Zaragoza, Spain
}

Submitted 14 April 2020; resubmitted 7 June 2020; accepted 2 August 2020;

first published online 2 April 2021

\begin{abstract}
Freight transport represents a very dynamic and competitive market with high requirements for reliability, lead time, cost, flexibility and visibility of transport service. Rationalization of transport service, reduced travelling time and reliable delivery times represent the main prerequisites for lowering the costs and increasing the efficiency of entire transport chain. These performance indicators actually represent the main factors affecting the shipper's mode choice. Improvement of these factors could be achieved by improved coordination between rail and non-rail-related stakeholders involved in freight transport service planning and realization. Since this solution requires a multi-stakeholder collaboration, it is needed to evaluate the interests of each of them in order to derive a preferred set of indicators, which will facilitate a collectively accepted solution and value alignment of all involved actors. In this paper, the preferred set of indicators was selected by using the DEcision MAking Trial and Evaluation Laboratory (DEMATEL) model technique integrated with the Analytic Network Process (ANP). DEMATEL is applied to analyse the causal relationships among the relevant dimensions and among the criteria within each dimension. The causal relationships are then used in ANP for determining the weights of the criteria. An empirical case study based on implementation of information sharing platform in rail intermodal transport chain is presented to demonstrate the effectiveness of the proposed approach. Based on this study the attributes that belong to reliability ("departing / arriving on-time", "cancelled services"), lead time ("idle time") and investment cost ("organizational culture", "business process redesign") dimensions represent five the most critical factors for obtaining a collectively accepted solution. This effective evaluation model enables policy makers and stakeholders in transportation / logistics to understand and conduct appropriate actions towards fulfilling the objectives for greener transportation.
\end{abstract}

Keywords: DEMATEL, ANP, rail intermodal transport chain, information sharing platform.

\section{Introduction}

Despite the initiatives made on European level for promoting the rail freight transportation as an environmentally friendly transport mode, a gap still exists between the needs and preferences of shippers on one side, and the offer of railway transport companies' on the other. Shippers request reliable, flexible and visible service with a minimum cost. Railway undertakings / operators still have difficulties to appropriately answer on these requests.

One of the innovative ways to improve rail freight service and to meet requested service criteria from a shipper's perspective is to improve the coordination between rail-related and non- rail-related stakeholders in transport chain. This can be achieved by developing a rail-enabled information sharing platform that will support planners in supply chain optimization and fulfil the shipper's requirements for real time visibility in whole transport chain. Some larger Logistics Service Providers (LSPs) have already implemented information sharing platforms. However, these are only partial solutions since they do not cover the whole intermodal transport chain. Specifically, there are difficulties to monitor the shipments during their travelling on railway legs due to non-availability of data. Existing rail-related information systems - "TIS RNE" (https://tis.rne.eu) for international train management and "Rail data" for exchange of information about movement of freight wagons - are community based and not open for non-railrelated stakeholders. This contributes to low visibility and consequently to low flexibility and reliability as well as to

${ }^{*}$ Corresponding author. E-mail: m.milenkovic@sf.bg.ac.rs

Copyright $\odot 2021$ The Author(s). Published by Vilnius Gediminas Technical University

This is an Open Access article distributed under the terms of the Creative Commons Attribution License (http://creativecommons.org/licenses/by/4.0/), which permits unrestricted use, distribution, and reproduction in any medium, provided the original author and source are credited. 
high lead times and costs of rail intermodal services and finally to lower competitiveness of railway transport mode.

In order to develop a rail-enabled information sharing platform it is needed to improve the data availability for rail transport. Since this innovation requires a joint effort and collective agreement by all stakeholders involved in the transport chain, for its successful implementation a multiple stakeholder collaboration is required. With a full collaboration among the stakeholders, smart interfaces between multiple stakeholders' data sources can be created to increase the quality of today's rail freight service and supply chain in general, making it more efficient, effective and sustainable.

Each of the involved stakeholders has its own role, interest and value added in the whole stakeholder network. However, interests of stakeholders are not fully aligned because their "environments" are not identical. In this paper, all actors who have the power to initiate the innovation are identified and the values they associate with the innovation are defined. These values represent specific dimensions within which there is a set of related attributes or performance indicators. In order to align their interests, it is needed to assess what dimensions / attributes are preferred by each stakeholder. It is also useful to understand the degree of influence of each dimension / attribute.

Therefore, the purpose of this study is to create a framework for decision making in case of developing and implementing a multi-value multi-stakeholder innovation in rail intermodal transport chain. To determine the level of significance of all effects and to calculate the level of their interdependences a combination of the two techniques, DEcision MAking Trial and Evaluation Laboratory (DEMATEL) and Analytic Network Process (ANP) is applied. DEMATEL method has been developed by Science and Human Affairs Program of the Battelle Memorial Institute of Geneva (Switzerland) between 1972 and 1976, and used for research and solving several groups of complicated and interdependent problems (Fontela, Gabus 1974, 1976).

In the context of this paper, DEMATEL provides a detailed analysis of causal relationships of dimensions / attributes by facilitating their separation into cause and effect groups. Derived causal relationships are then used to determine the weights of the attributes by applying the ANP method. ANP method represents an improved version of Analytic Hierarchy Process (AHP) method (Saaty 2008). It is designed to avoid hierarchical limitations of the AHP method. Evaluation is performed from the aspect of each stakeholder separately. In order to derive the final ranking of attributes the relative weight of involved stakeholders is considered.

The paper is organized as follows. After the introduction, in Section 1 a comprehensive literature review is given. Section 2 describes the methodology based on integration of DEMATEL and ANP approaches for selection a set of indicators, which is collectively accepted by the most important stakeholders in the transport chain.
Section 3 describes the application of the approach in case of implementing an information sharing platform in rail intermodal transport. Concluding remarks and directions for further research are given in the last section.

\section{Literature review}

In this section, the most relevant related literature is reviewed. The section is divided on two parts. In Subsection 1.1 contributions related to the selection of the main underlying factors that determine the freight transport service competitiveness are discussed. Applications of DEMATEL-ANP approach in transport environment are described in Subsection 1.2.

\subsection{Competitiveness of freight transport service offer}

There are numerous factors that determine the competitiveness of rail freight transport and consequently the shippers' mode choice. Some of these factors are related to macroeconomic and structural attributes (Gleave et al. 2015). In addition, de-industrialization and fragmentation of logistics processes can be considered as macro level factors, which limit the attractiveness of rail transport from shipper's perspective (Gölcük, Baykasoğlu 2016). However, encouraging mode shift through targeting the key factors affecting the competitiveness of rail freight may have a significant impact on shipper's mode choice. Table 1 summarizes the most relevant contributions dealing with the analysis of transport performance indicators.

Danielis et al. (2005) conducted an analysis about logistics managers' preferences for freight service attributes based on an experiment performed in two Italian regions. Results highlight the strong preference for attributes of quality (time, reliability and safety) over cost. García-Menéndez et al. (2006) analysed the determinants of mode choice decisions for Spanish shipments to Europe and North Africa. Analysis has revealed that the quality of service attributes influences modal choice for high-value commodity flows whereas the transport costs are the main factors for low-value commodity flows. According to Patterson et al. (2007), a shipper chooses the carrier according to a number of attributes that characterize the shipper (firm size, location, accessibility), the shipment (type, size / weight, value, perishability), the distance and the carrier. As the most important carrier, related attributes the authors highlight total shipment costs, delivery time, service reliability, service flexibility, service frequency and the quality of customer service.

On-time reliability was found to strongly increase the probability of choosing a carrier. The study concluded that increasing the rail share of freight transportation would require a change in reputation as well as improvements in the standard attributes. Samimi et al. (2010) proposed an activity based microsimulation framework for freight modelling. According to the results of the analysis, use of road is more sensitive to travel time than the use of rail. 
Table 1. Summary of the most relevant contributions dealing with analysis of transport performance indicators

\begin{tabular}{|c|c|c|c|}
\hline Reference & Problem type & Attributes considered & Main findings \\
\hline $\begin{array}{l}\text { Danielis et al. } \\
\text { (2005) }\end{array}$ & $\begin{array}{l}\text { estimation of logistics } \\
\text { managers' preferences for } \\
\text { freight service attributes }\end{array}$ & $\begin{array}{l}\text { "» freight cost; } \\
\text { "» travel time; } \\
\text { "»" risk of delay; } \\
\text { "» risk of loss and damage }\end{array}$ & $\begin{array}{l}\text { strong preference for quality attributes } \\
\text { (reliability) over the cost attributes }\end{array}$ \\
\hline $\begin{array}{l}\text { García-Menéndez } \\
\text { et al. (2006) }\end{array}$ & $\begin{array}{l}\text { analysis of determinants } \\
\text { of mode choice decisions }\end{array}$ & $\begin{array}{l}\text { "»" cost of transport; } \\
\text { "» transit time; } \\
\text { "» service frequency; } \\
\text { "»" distance; } \\
\text { "»" damage and losses; } \\
\text { "» delays; } \\
\text { "»" consolidation and environmental restric- } \\
\text { tions for lorries to circulate at week-ends }\end{array}$ & $\begin{array}{l}\text { the quality of service attributes } \\
\text { influences modal choice for high- } \\
\text { value commodity flows whereas the } \\
\text { transport costs are the main factors for } \\
\text { low-value commodity flows }\end{array}$ \\
\hline $\begin{array}{l}\text { Patterson et al. } \\
(2007)\end{array}$ & $\begin{array}{l}\text { analysis of shippers mode } \\
\text { choice conducted for a } \\
\text { Canadian freight corridor }\end{array}$ & $\begin{array}{l}\text { "» shipper and } \\
\text { "»" shipment attributes; } \\
\text { "» geographic and time characteristics; } \\
\text { "» carrier attributes }\end{array}$ & $\begin{array}{l}\text { on-time reliability was found to } \\
\text { strongly increase the probability } \\
\text { of choosing a carrier; a change in } \\
\text { reputation as well as improvements in } \\
\text { the standard attributes are needed for } \\
\text { increasing the rail share }\end{array}$ \\
\hline $\begin{array}{l}\text { Samimi et al. } \\
(2010)\end{array}$ & $\begin{array}{l}\text { framework for } \\
\text { behavioural freight } \\
\text { movement modelling }\end{array}$ & $\begin{array}{l}\text { "»" transit time; } \\
\text { "»" reliability; } \\
\text { "» shipment size and frequency; } \\
\text { "» use of Information Technology (IT); }\end{array}$ & $\begin{array}{l}\text { use of road is more sensitive to } \\
\text { travel time than the use of rail; larger } \\
\text { shipments are more likely to be } \\
\text { transported by rail }\end{array}$ \\
\hline $\begin{array}{l}\text { Brooks et al. } \\
(2012)\end{array}$ & $\begin{array}{l}\text { assessment of freight } \\
\text { shippers' preferences for } \\
\text { services }\end{array}$ & $\begin{array}{l}\text { "»" frequency; } \\
\text { "» transit time; } \\
\text { "»" freight distance; } \\
\text { "»" direction (headhaul / backhaul); } \\
\text { "» reliability measured by delivery window; } \\
\text { ""» reliability measured by delay and price }\end{array}$ & $\begin{array}{l}\text { confirmed presence of trade-offs } \\
\text { by shippers involving costs and } \\
\text { benefits of reducing transit time, } \\
\text { improving on-time arrival reliability } \\
\text { and mitigating the risk of long arrival } \\
\text { delays }\end{array}$ \\
\hline Combes (2012) & $\begin{array}{l}\text { an inventory-theoretical } \\
\text { model of optimal } \\
\text { shipment size }\end{array}$ & $\begin{array}{l}\text { "»" transportation cost; } \\
\text { "»" shipment size; } \\
\text { "»" travel time; } \\
\text { "»" commodity flow rate between the ship- } \\
\text { per and receiver }\end{array}$ & $\begin{array}{l}\text { the rate of the commodity flow to } \\
\text { which the shipment belongs impacts } \\
\text { on the shipment size, which depends } \\
\text { on the transportation mode, and } \\
\text { the freight mode choice depends on } \\
\text { shipment size as well }\end{array}$ \\
\hline $\begin{array}{l}\text { Moschovou, } \\
\text { Giannopoulos } \\
(2012)\end{array}$ & $\begin{array}{l}\text { assessment of users' } \\
\text { perception in the freight } \\
\text { transportation system }\end{array}$ & $\begin{array}{l}\text { "» reliability of the service (in terms of trav- } \\
\text { el times, simplicity of procedures); } \\
\text { "»" value of the load; } \\
\text { "»" size of the load; transportation cost; } \\
\text { "»" shipment life; } \\
\text { "»" capability of tracking and tracing; } \\
\text { "» availability of the loading and unloading } \\
\text { equipment }\end{array}$ & $\begin{array}{l}\text { from the aspect of both, the shipment } \\
\text { owners and forwarders, the reliability } \\
\text { of service is one of the most important } \\
\text { attributes }\end{array}$ \\
\hline Kim et al. (2017) & $\begin{array}{l}\text { analysis of shippers' } \\
\text { mode choice preference } \\
\text { heterogeneity }\end{array}$ & $\begin{array}{l}\text { "»" price; } \\
\text { "» service time; } \\
\text { "» reliability; } \\
\text { "» flexibility; } \\
\text { "» modal connectivity; } \\
\text { "» intermodal transfer; specialised handling; } \\
\text { "»" value added activities; } \\
\text { "»" environmental and sustainability issues }\end{array}$ & $\begin{array}{l}\text { the time and reliability are the most } \\
\text { important factors for shippers }\end{array}$ \\
\hline $\begin{array}{l}\text { Solakivi, Ojala } \\
(2017)\end{array}$ & $\begin{array}{l}\text { analysis of the key } \\
\text { determinants of carrier } \\
\text { selection }\end{array}$ & $\begin{array}{l}\text { "»" cost; } \\
\text { "» reliability; } \\
\text { "»" service level; } \\
\text { "» information sharing capabilities of the } \\
\text { service provider; } \\
\text { "»" environmental issues }\end{array}$ & $\begin{array}{l}\text { service perceptions represent one of } \\
\text { the key variables for mode and carrier } \\
\text { selection }\end{array}$ \\
\hline Shin et al. (2019) & $\begin{array}{l}\text { analysis of characteristics } \\
\text { of freight mode choices } \\
\text { made by shippers } \\
\text { and carriers with the } \\
\text { introduction of a new } \\
\text { freight transport system }\end{array}$ & $\begin{array}{l}\text { "»" transport time; } \\
\text { "»" service level; } \\
\text { "» transport cost }\end{array}$ & $\begin{array}{l}\text { reduction of the transport cost is } \\
\text { more effective than the reduction of } \\
\text { the transport time or increasing the } \\
\text { service level for successful acceptance } \\
\text { of a new freight transport system }\end{array}$ \\
\hline
\end{tabular}


Shipment size/ weight emerged as a significant variable, indicating that larger shipments are more likely to be transported by rail. Brooks et al. (2012) analysed the Australian domestic freight transport market and assessed the freight shippers' preferences for services offered by freight transportation providers. Seven service attributes were considered: frequency, transit time, freight distance, direction (headhaul/backhaul), reliability measured by delivery window, reliability measured by delay and price. The analysis confirmed the presence of trade-offs by shippers involving costs and benefits of reducing transit time, improving on-time arrival reliability and mitigating the risk of long arrival delays. Based on the analysis of preferences of 3000 shippers in France the frequency of service also appeared as an important factor in mode choice. Combes (2012) empirically validates an inventorytheoretical model of optimal shipment size. Validation is based on a large and heterogeneous population of shipments. It is concluded that the shipment size depends on transportation mode, and that the freight mode choice depends on shipment size as well. The rate of the commodity flow to which the shipment belongs plays also an important role in determining the shipment size and mode choice. Moschovou and Giannopoulos (2012) conducted a research study in case of freight market in Greece in order to develop the mechanisms and factors that influence freight transport mode choice and to design the mathematical models relating to users' perception of the system with a set of relevant criteria and parameters. The modelling was done from the point of view of the owners of the goods as well as the forwarders / LSPs considering two perspectives: the importance as well as the satisfaction received. Included attributes were: reliability of the service (in terms of travel times, simplicity of procedures), value of the load, size of the load, transportation cost, shipment life, capability of tracking and tracing, availability of the loading and unloading equipment. Summary of the results from regression analysis has shown that in both perspectives for both, the owners and the forwarder, the reliability of the service was among the most important attributes. Kim et al. (2017) applied the latent class modelling in order to improve the understanding of the decision-making process of freight shippers in New Zeeland when they make a mode choice decision. The analysis has shown that the time and reliability are the most important factors for shippers. Solakivi and Ojala (2017) has made an extensive carrier selection literature review illustrating how the key determinants of carrier selection have evolved during the years. A critical view is on the carrier selection process is made by using survey methodology and an improved survey tool was suggested. Besides the traditional determinants such as cost, reliability and service level, information sharing capabilities of the service provider and environmental issues were also considered. According to Shin et al. (2019) Korean freight transport market is characterized by increased structural problems due to an extremely high proportion of road transport in the freight transport process. The authors consider changes in the Korean freight transport system assuming the introduction of a new intermodal automated freight transport system and performed a stated preference survey to identify changes in the perception of the shippers and carriers in the freight transport market. The attribute variables included in the survey were transport time, service level and transport cost. By analysing the results of the developed mode choice model based on the introduction of new freight transport system, authors concluded that for the shift of the demand to the new transport mode the strategies for reducing the transport cost are more effective than the strategies for reducing transport time or increasing the service level.

\subsection{Application of DEMATEL and ANP in transportation sector}

DEMATEL and ANP approaches are frequently used in literature for solving Multi-Criteria Decision-Making (MCDM) problems. Recently, Büyüközkan and Güleryüz (2016) made a detailed review of the applications of DEMATEL and ANP in different business areas. Here, the analysis is limited to the contributions related to transport environment. Table 2 summarizes the most relevant applications according to the problem type and modality. Besides the combined DEMATEL-ANP, review includes also important contributions based on combination of DEMATEL and/or ANP with other advanced MCDM techniques.

Liou et al. (2007) proposed a hybrid MCDM model to address dependent relationships among criteria for airline safety systems. Ten criteria divided in four dimensions were evaluated. An empirical testing of the approach using a Taiwanese case study illustrated the applicability of the approach. Lin et al. (2010) studied the problem of identifying the required VTS utilities between distinguishing characteristics / features of consumers and proposed the ideal service combinations for the next e-era generation VTS. DEMATEL is used to construct the Network Relation Map (NRM) among the criteria of each aspect. Then ANP-based on NRM is used to determine relative weightings among those criteria. Technique for Order Preference by Similarity to Ideal Solution (TOPSIS) is used to determine and improve the gaps among the distinguishing characteristics / features of consumers' desired utilities. Vujanović et al. (2012) weight of indicators used in fleet maintenance management. Proposed model has been tested in case of several companies with road vehicle fleets. Hsu and Liou (2013) applied DEMATEL-ANP for the selection of outsourcing providers in the airline sector. The developed model is implemented using data from a Taiwanese airline operator. Eleven partner selection criteria belonging to four dimensions were considered. Liu et al. (2013) analysed different strategies for improvement of connection service to the urban airport in Taipei. Authors proposed a hybrid MCDM method to address the dependent relationships among the ten criteria, which belong to three dimensions. First, the DEMATEL was applied in 
Table 2. Main characteristics of relevant DEMATEL and ANP applications in transportation / logistics domain

\begin{tabular}{|c|c|c|}
\hline Reference & Problem type & Modality \\
\hline Liou et al. (2007) & selection of criteria for airline safety measurement & airline \\
\hline Lin et al. (2010) & identifying the required Vehicle Telematics Systems (VTS) requirements & road \\
\hline Vujanović et al. (2012) & evaluation of indicators for fleet maintenance management & road \\
\hline Hsu, Liou (2013) & selection of outsourcing providers in airline sector & airline \\
\hline Liu et al. (2013) & strategies for improvement of connection service to the urban airport & airline \\
\hline Kundakc1 et al. (2014) & cargo shipping company selection problem & intermodal \\
\hline Liou et al. (2014) & service quality improvement of public transport systems & road \\
\hline Pamučar, Ćirović (2015) & acquisition of forklifts in logistics centers & intermodal \\
\hline Chen (2016) & airline service quality improvement & airline \\
\hline Ranjan et al. (2016) & evaluation of performances of different railway zones & rail \\
\hline Bongo, Ocampo (2017) & selection of air traffic flow management actions to mitigate airport congestion & airline \\
\hline Dinçer et al. (2017) & balanced scorecard approach for evaluating the performance of European airlines & airline \\
\hline Ha, Yang (2017) & analysis of Port Performance Indicators (PPIs) & maritime \\
\hline Ha et al. (2017) & analysis of PPIs taking into account multiple objectives of stakeholders & maritime \\
\hline Zhan et al. (2017) & railway accidents analysis & rail \\
\hline Bongo et al. (2018) & air traffic controllers' workload stress problem & airline \\
\hline Hsu et al. (2018) & quality of service for bike sharing systems & bicycling \\
\hline Kijewska et al. (2018) & evaluation of measures for sustainable urban freight transport & road \\
\hline Lee et al. (2018) & optimal green aviation fleet management & airline \\
\hline Lu et al. (2018) & airport sustainability problem & airline \\
\hline Gudiel Pineda et al. (2018) & improvement of airline performance & airline \\
\hline Shaik, Abdul-Kader (2018) & performance measurement in reverse logistics & intermodal \\
\hline Yazdani et al. (2020) & evaluation of performances of freight transportation companies & general \\
\hline Karaşan, Kahraman (2019) & selection of freight villages location & intermodal \\
\hline Kumar, Anbanandam (2020) & assessment of the main inhibitors in intermodal railroad transport & intermodal \\
\hline
\end{tabular}

order to construct the NRM, and then the relative weights of the criteria were assessed by DEMATEL-ANP and in the last phase, improvement priority was determined by using the multi-criteria optimization and compromise solution (in Serbian - Višekriterijumska optimizacija I KOmpromisno Rešenje - VIKOR) method. Kundakcı et al. (2014) proposed a MCDM model that combines DEMATEL and ANP for cargo shipping company selection problem. DEMATEL is employed to compute the effects between selection criteria and ANP is used to determine the best cargo shipping company. Liou et al. (2014) assessed the potential options to improve the service quality of public transportation systems. DEMATEL technique was first applied to assess the relations between dimensions and criteria. Subsequently, influential weights were calculated by DEMATEL-ANP. In order to aggregate, the weighted gaps the authors applied an information fusion technique. Proposed model was tested on a real case study of Taipei city bus companies. Pamučar and Ćirović (2015) proposed an application of the DEMATEL-MABAC (Multi-Attributive Border Approximation Area Comparison) model in the process of making investment decisions on the acquisition of forklifts in logistics centers. Weight coefficients of criteria were obtained based on DEMATEL method. The evaluation and selection of forklifts was car- ried out using MABAC method. Chen (2016) applied DEMATEL-ANP approach to select airline service quality improvement criteria for the Taiwanese airline industry. The study provides airlines with a direction for measuring and improving their service quality with the goal of developing sustained competitive advantage over the long term. Ranjan et al. (2016) proposed a combined methodology for appraising and evaluation the performances of different railway zones of Indian Railways. In this approach, causal relationships between selected criteria are evaluated by DEMATEL. A compromise ranking of different railway zones with respect to a set of evaluation criteria is derived employing VIKOR method. Authors demonstrated a practical usability of proposed combined methodology for evaluating the performances of railway zones. Bongo and Ocampo (2017) developed an integrated fuzzy MCDM model to mitigate the airport congestion. The developed model integrates the concepts of DEMATEL, TOPSIS and fuzzy set theory under a fuzzy environment to extract the inherent relationships of criteria considered as well as to evaluate and select the most suitable air traffic flow management action that can mitigate airport congestion. The applicability of proposed approach is demonstrated in a case study at Ninoy Aguino international airport. Dinçer et al. (2017) evaluated the performance of the European 
airlines using a balanced scorecard perspective. Proposed approach is based on fuzzy DEMATEL, fuzzy ANP and Multi-Objective Optimization on the basis of Ratio Analysis (MOORA) methods. As the output of proposed approach, the set of recommendations was derived for the European airline companies to improve their performances. Ha and Yang (2017) compared the analysis of PPIs importance considering their independent relationship using an AHP and their interdependent relationship using a hybrid approach based on DEMATEL and ANP. The results demonstrate a similar variance of relative importance across the PPIs but a clear difference on their importance scores and ranking. Ha et al. (2017) developed a framework based on the combination of DEMATEL and ANP together with Fuzzy Evidential Reasoning (FER) to assess the interdependency among PPIs and to incorporate multiple objectives of key stakeholders. Authors identify the overall PPIs with respect to different stakeholders as well as evaluate the weights of the interdependency PPIs and synthesize the evaluation of quantitative and qualitative PPIs with their weights through an intelligent decision support tool. Proposed approach was tested on a case of four major South Korean container ports. Zhan et al. (2017) developed a hybrid human and organizational analysis method for railway accidents based on ANP combined with fuzzy DEMATEL. Specifically, to find out the leading causes of the accident, an ANP method is applied. The ANP method clarifies causal relationships of causes. Fuzzy DEMATEL technique is adopted to conduct the inner dependency analysis. Bongo et al. (2018) addressed controllers' workload stress problem by identifying the main stressors and ranking the probable measures, which can be applied for solving this issue. Combined DEMATEL-ANP and Preference Ranking Organization METHod for Enrichment Evaluation (PROMETHEE) II approach is implemented for the problem in a case study of Mactan Civil Aviation Authority of the Philippines. Hsu et al. (2018) proposed a DEMATEL-ANP approach for assessment of dependent relationships between various criteria for the quality of service for bike-sharing systems. A modified VIKOR method is applied to explore the weighted gap to the aspiration levels. In order to handle ambiguity related in questionnaire responses and to incorporate the uncertainty of human thinking in the evaluation, authors applied grey theory. Developed hybrid MCDM model that combines the Grey-DANP (Grey DEMATEL-based ANP) and Grey-VIKOR methods is tested using data for Taipei's Youbike and Obike systems. Kijewska et al. (2018) applied a DEMATEL-AHP approach in analysing the set of measures for sustainable urban freight transport. Diverse expectations of the main stakeholder groups were included by Multi-Actor MultiCriteria Analysis (MAMCA). Proposed approach was tested on a case of Szczecin Municipality (Poland) during the implementation of a sustainable urban freight transport system in the city area. Lee et al. (2018) proposed a MCDM approach, which integrates the DEMATEL, ANP and $0-1$ goal programming to achieve optimal green avia- tion fleet management strategy decisions. Integrated model helps airlines project managers to accurately understand how to allocate resources and obtain the final optimal portfolio for a sustainable green aviation fleet when resources are limited. Lu et al. (2018) examined the airport sustainability problem and recommended an approach for estimation and selection of criteria for sustainable performance using sustainability-balanced scorecard approach. Proposed model is based on a DEMATEL-ANP for prioritizing the 15 criteria grouped in 5 perspectives. Then, a hybrid modified VIKOR is used to select and improve the performance gaps between the aspiration values and the current situation for the international airport. Gudiel Pineda et al. (2018) proposed an integrated model that combines data mining and MCDM to extract the critical factors for the improvement of airline performance. Dominance-based rough set approach is applied to extract the essential factors. DEMATEL-ANP is then used to construct the complex evaluation system. VIKOR method is applied to select suitable improvement alternative goals. Shaik and Abdul-Kader (2018) proposed a hybrid MCDM approach for performance measurement of reverse logistics. Proposed approach combines DEMATEL, fuzzy ANP and AHP methods. Developed performance evaluation model incorporates relevant attributes and contributes to a more realistic representation of the enterprise's performance by calculating the overall comprehensive performance by calculating the overall comprehensive performance index. Yazdani et al. (2020) proposed a decision analysis tool that integrates rough number based DEMATEL and MABAC methods for evaluating the performances of freight transportation companies. The authors proved that the proposed rough set based decisionmaking approach can be very useful tool for facilitating sustainability measurement in freight transportation. Karaşan and Kahraman (2019) proposed an integrated fuzzy decision model for the selection of freight villages' location. Authors applied DEMATEL for determining the most effective criteria as well as their inner and outer dependencies. ANP was used for weighting the determined criteria and TOPSIS for finding the best location alternative. Kumar and Anbanandam (2020) proposed a hierarchical framework based on Grey-DANP to assess the interdependency and priority of the main inhibitors in intermodal railroad transport. Authors considered operational, technological and infrastructural, knowledge, organisational and government policy and regulations dimensions and its attributes for evaluating the intermodal railroad inhibitors. Proposed framework is validated with a case of the Indian railroad system.

\subsection{Research gap and contribution of this paper}

As it can be noticed from the discussion of previous research, our study is the first attempt to assess the potential for development and implementation of an innovation in rail intermodal transportation taking into account 
a multi-value multi-stakeholder perspective. Given the broad set of attributes we are considering for this analysis as well as the different perspective of every stakeholder involved, the approach introduced in this paper is the most comprehensive of any existing approaches for the assessment of critical attributes for successful implementation of a multi-value multi-stakeholder innovation. One of the main causes of unsuccessful implementation of innovations in freight transportation is the lack of collective acceptance and value misalignment of involved actors. In response to this research challenge, we proposed a methodology, which may help decision makers to reduce the risk of failed implementation of innovations.

\section{Methodology}

A MCDM model is proposed, which combines DEMATEL and ANP to assess the effect of each dimension and attribute and to measure the importance of each attribute from the aspect of each actor involved in rail intermodal transport chain.

\subsection{Data collection}

Any innovation in transport sector is complex in nature and involves multiple stakeholders with different roles, backgrounds and values. A clear picture about the value network, all stakeholders involved and their interrelationships was made based on a number of interviews with the experts and stakeholders. Innovations provide substantial benefits for final customers (consignors and consignees) through lower transport costs, higher performances of transport services and greener supply chain, however they are not included into the analysis since they do not have the influence to initiate the innovation. In case of rail-en- abled information sharing platform following actors are included in the analysis:

"» shippers / manufacturers;

"» LSPs;

"» terminal operators;

"» railway undertakings / railway operators;

") railway infrastructure managers;

»» rolling stock leasing companies.

The value network map that represents the relationships between the involved value chain actors is given on Figure 1. The value network map displays the resources that actors exchange or pool as part of their operations (information, mobile capacities, infrastructure or financial assets). Information on exchange resources among actors is qualitative - the map illustrates that two actors exchange money and information but not the exact quantities. However, these key resources are of strategic type and they are considered as underlying valuable resources achieved from the relationship among the stakeholders within the rail-enabled information sharing solution. Financial exchanges are implicitly included through the service provisions between stakeholders. It can be noticed that the presented network represents a very complex value chain, considering that stakeholders in it may feed into a number of different value chains (Kaplinsky, Morris 2001).

Based on an extensive literature review and discussions with experts within the Smart Rail project (SmartRail 2016) and Clusters 2.0 (2019) project, the sets of dimensions and attributes are defined.

Dimensions are related to the set of values that stakeholders and experts associate with rail-enabled information sharing platform. Provision of information in a real time results in a higher quality of rail freight service due to the fact that the sharing of information enables users of the platform to provide better services to the end clients.

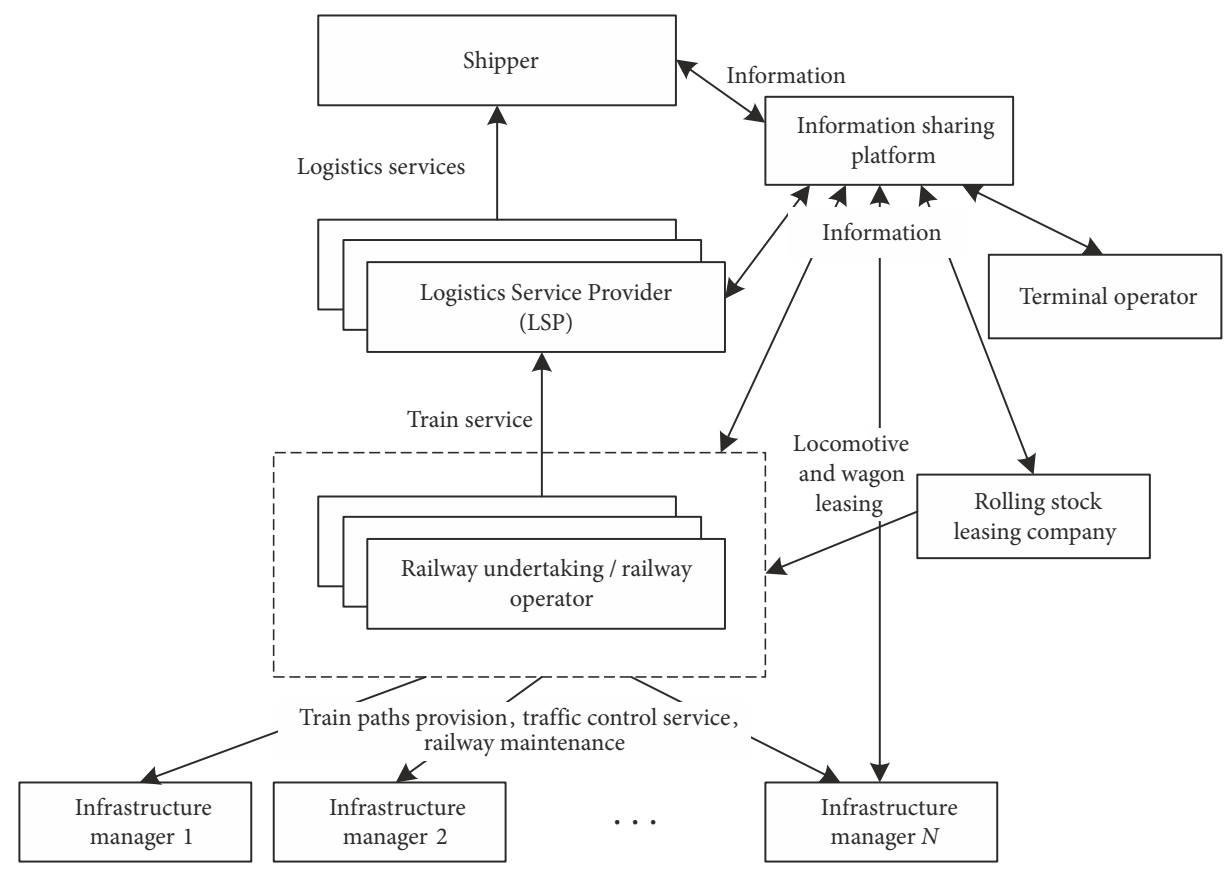

Figure 1. Value network map - rail intermodal transport chain 
Therefore, innovation in information sharing platform is positively correlated with:

"») reliability - increased predictability as a result of better management of expectations will result in increased reliability;

"»» visibility - improved tracking and tracing of shipments will result in increased visibility;

"» lead time - increased information flow leads to a reduced lead time; increased predictability will also contribute to stability of lead time;

"» efficiency of service provision - intensive information sharing will contribute to improved efficiency of resource (fixed, mobile, human) utilization and consequently decrease the cost per transport unit, which will enable to offer more competitive price on rail;

"' flexibility - intensive information sharing enables to actors a higher ability to book or rebook a slot on a train, in terminal or warehouse;

"» revenue - through higher reliability, visibility, shorter lead time, higher efficiency (reduced transport service cost) railway operators as well as the LSPs, shippers and terminal operators will be able to increase revenues;

"») cost of investing in innovation - development and implementation of information sharing platform requires a joint investment of all stakeholders. Besides the direct costs related to the platform implementation, there are also the costs for organizational redesign in order to make a shift from traditional towards more supply chain-oriented organization.

The set of indicators for each of the five aspects or dimensions related to innovations in rail intermodal transportation is given in Table 3.

The set of performance indicators listed in Table 1 was distributed in the form of a Stage 1 questionnaire to the experts in order to define the most important subset of criteria. Preferences regarding the attribute dimensions were also the subject of Stage 1 questionnaire. In total 25 responses were obtained in the period from July to September 2019. Respondents were five university teachers, three researchers from eminent research institutes, and by three representatives of big shippers, LSPs, terminal operators, railway undertakings / operators and infrastructure managers and two representatives of rolling stock leasing companies. Expert's evaluations in both cases (determining the most important subset of criteria as well as the subset of the most preferred dimensions for every stakeholders) ranged from 0 to 10 , with a high score meaning high importance of the criteria / dimension (Figure 2).

In order to obtain a preferred subset of criteria / dimension for each stakeholder Triangular Fuzzy Numbers (TFNs) were used. TFNs represent triplets ("a", "b", “c"). First and third numbers of the triplet " $a$ " and "c" represent left and right spread respectively, whose membership functions are 0 , whereas the number "b" denotes the mean value of the fuzzy number with membership function value equal to 1 (Pamučar et al. 2012). Preferred subset of criteria based on TFNs (with a mean of 7.5 and above) is shown in Table 4. Preferred dimensions for each of the stakeholders are given in Section 3.

Compiled results of Stage 1 were used in Stage 2 for the design of questionnaire based on the DEMATEL and ANP. The questionnaire aimed at evaluation of interdependency and impact between dimensions and attributes for each stakeholder. In total 58 surveys were collected within the period from October to December 2019. Responses were obtained through personal interview and completed surveys from five university professors, five researchers from eminent research institutes, and by eight representatives of big shippers, seven representatives of LSPs, seven representatives of terminal operators, ten representatives of railway undertakings / operators and by eight representatives of infrastructure managers and rolling stock leasing companies. The network structure for prioritizing and weighing of dimensions and attributes is illustrated on Figure 3. Stakeholders ranking does not include mutual influence of different stakeholder. On the level of attributes interrelationships within (dotted lines) and between (dashed lines) dimensions are considered.

\subsection{DEMATEL method}

The aim of the DEMATEL is to assess the level of interdependencies that exist between selected attributes as well as to construct a NRM. More specifically, we applied DEMATEL method in order to estimate the direction and intensity of interlinks between selected dimensions and attributes that correlate with the implementation of railenabled information sharing platform (Vujanović et al. 2012). DEMATEL method is based on the perception of individuals. The essence of DEMATEL technique is a matrix account applied for determining the intensity of direct and indirect relationships and impact intensity (Chen et al. 2011; Ranjan et al. 2015; Yazdani et al. 2017). The method begins with the establishment of a matrix, which represents the direct impact between pairs of dimensions / attributes. Normalized direct relation matrix serves for computation of total relation matrix by applying a set of matrix operations. Total relation matrix includes both direct and indirect influence of the dimensions or attributes (Quezada et al. 2018). The output of the DEMATEL process is a NRM, which illustrates the interrelation between dimensions / attributes and identifies what is central to the problem as well as which dimensions / attributes affect each other or themselves (Chen et al. 2011). Details of the procedure for calculating the level of interdependence of the considered factors by DEMATEL are given in Appendix.

\subsection{Integrated DEMATEL and ANP}

In order to evaluate the effects of an innovation development and implementation in a complex multi-stakeholder environment it is needed to consider multiple attributes and determine the relative priorities of these attributes. 
Table 3. Railway freight transport - performance evaluation factors (Smart-Rail 2016)

\begin{tabular}{|c|c|}
\hline Dimension & Criteria \\
\hline \multirow{7}{*}{ Reliability } & departing / arriving on-time or within defined tolerance \\
\hline & cancelled services \\
\hline & train path availability (\% of successful satisfaction vs. rejections) \\
\hline & average delay of a train \\
\hline & railway infrastructure disrupting events and their impact \\
\hline & time deviation of the demanded train path (demanded vs. assigned) \\
\hline & average delay of the loading / unloading process in terminal \\
\hline \multirow{7}{*}{ Visibility } & cargo for which the information in transit are available \\
\hline & mileage on which the information in transit are available \\
\hline & availability of precise tracking and tracing \\
\hline & availability of information in case of disruptions \\
\hline & idle time between arising an event and getting informed \\
\hline & visibility of the alternate or diverted route \\
\hline & data exchange standards supported by the Information and Communications Technology (ICT) systems \\
\hline \multirow{9}{*}{ Flexibility } & number of departures per day and per destination \\
\hline & destinations served by the terminal or by the whole network \\
\hline & frequency of the first mile (last mile) service \\
\hline & additional capacity available on the existing train services \\
\hline & availability of an additional service in case of special needs (e.g., additional train load) \\
\hline & the time necessary to book a slot on a train \\
\hline & cut-off time - when the transport unit needs to come to the terminal \\
\hline & ability to rebook or change transport (its destination or parameters) \\
\hline & the time necessary to rebook or change \\
\hline \multirow{7}{*}{ Lead time } & average time necessary to load / unload a train in a terminal or in an end point \\
\hline & average time necessary to get wagons coupled to form a train \\
\hline & average idle time (waiting in terminal, waiting for departure, for handling in port) \\
\hline & waiting for equipment (time to wait for assigning equipment or resources (engine, driver) \\
\hline & waiting for another train to assume the wagons / cargo) \\
\hline & handover time between the partners \\
\hline & various other services (technical inspections, customs, commercial inspections, etc.) \\
\hline \multirow{9}{*}{$\begin{array}{l}\text { Efficiency } \\
\text { of service } \\
\text { provision }\end{array}$} & utilization of train capacity \\
\hline & utilization of engine power \\
\hline & percentage of empty wagon / container runs \\
\hline & number of cancellations of train service \\
\hline & total transit costs (terminal to terminal, door to door) \\
\hline & time for loading / unloading at terminals \\
\hline & utilization rate of the marshalling yard \\
\hline & volume of data exchange (no of different messages / processes / communication dialogs covered by data exchange) \\
\hline & productivity of terminal facilities \\
\hline \multirow{5}{*}{ Revenue } & market share \\
\hline & revenue per train \\
\hline & revenue per leg \\
\hline & creating more possibilities (e.g., by cooperation) \\
\hline & risk on missing revenues (e.g., by cooperation) \\
\hline \multirow{5}{*}{$\begin{array}{l}\text { Investment } \\
\text { cost }\end{array}$} & data sharing systems \\
\hline & human resources \\
\hline & organizational culture (mental shift) \\
\hline & business processes redesign \\
\hline & equipment and facilities for efficient distribution of shipments \\
\hline
\end{tabular}


Table 4. Attributes with a mean of 7.5 and above

\begin{tabular}{|c|c|}
\hline Dimension & Criteria \\
\hline \multirow{2}{*}{ Reliability (A) } & departing / arriving on-time or within defined tolerance $a_{1}$ \\
\hline & cancelled services $a_{2}$ \\
\hline \multirow{3}{*}{ Visibility (B) } & availability of precise tracking and tracing $b_{1}$ \\
\hline & availability of information in case of disruptions $b_{2}$ \\
\hline & data exchange standards supported by the ICT systems $b_{3}$ \\
\hline \multirow{5}{*}{ Flexibility (C) } & number of departures per day and per destination $c_{1}$ \\
\hline & destinations served by the terminal or by the whole network $c_{2}$ \\
\hline & additional capacity available on the existing train services $c_{3}$ \\
\hline & ability to rebook or change transport (its destination or parameters) $c_{4}$ \\
\hline & the time necessary to rebook or change $c_{5}$ \\
\hline \multirow{2}{*}{ Lead time (D) } & average time necessary to load / unload a train in a terminal or in an end point $d_{1}$ \\
\hline & average idle time (waiting in terminal, waiting for departure, for handling in port) $d_{2}$ \\
\hline \multirow{5}{*}{$\begin{array}{l}\text { Efficiency of service } \\
\text { provision (E) }\end{array}$} & utilization of train capacity $e_{1}$ \\
\hline & percentage of empty wagon / container runs $e_{2}$ \\
\hline & total transit costs (terminal to terminal, door to door) $e_{3}$ \\
\hline & productivity of terminal facilities $e_{4}$ \\
\hline & $\begin{array}{l}\text { volume of data exchange (no of different messages / processes / communication dialogs covered by data } \\
\text { exchange) } e_{5}\end{array}$ \\
\hline \multirow{2}{*}{ Revenue (F) } & market share $f_{1}$ \\
\hline & revenue per train $f_{2}$ \\
\hline \multirow{3}{*}{ Investment cost $(\mathrm{G})$} & data sharing systems $g_{1}$ \\
\hline & business processes redesign $g_{2}$ \\
\hline & organizational culture (mental shift) $g_{3}$ \\
\hline
\end{tabular}

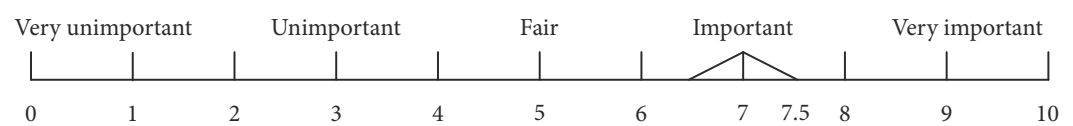

Figure 2. Rank of importance level (Chen et al. 2011)

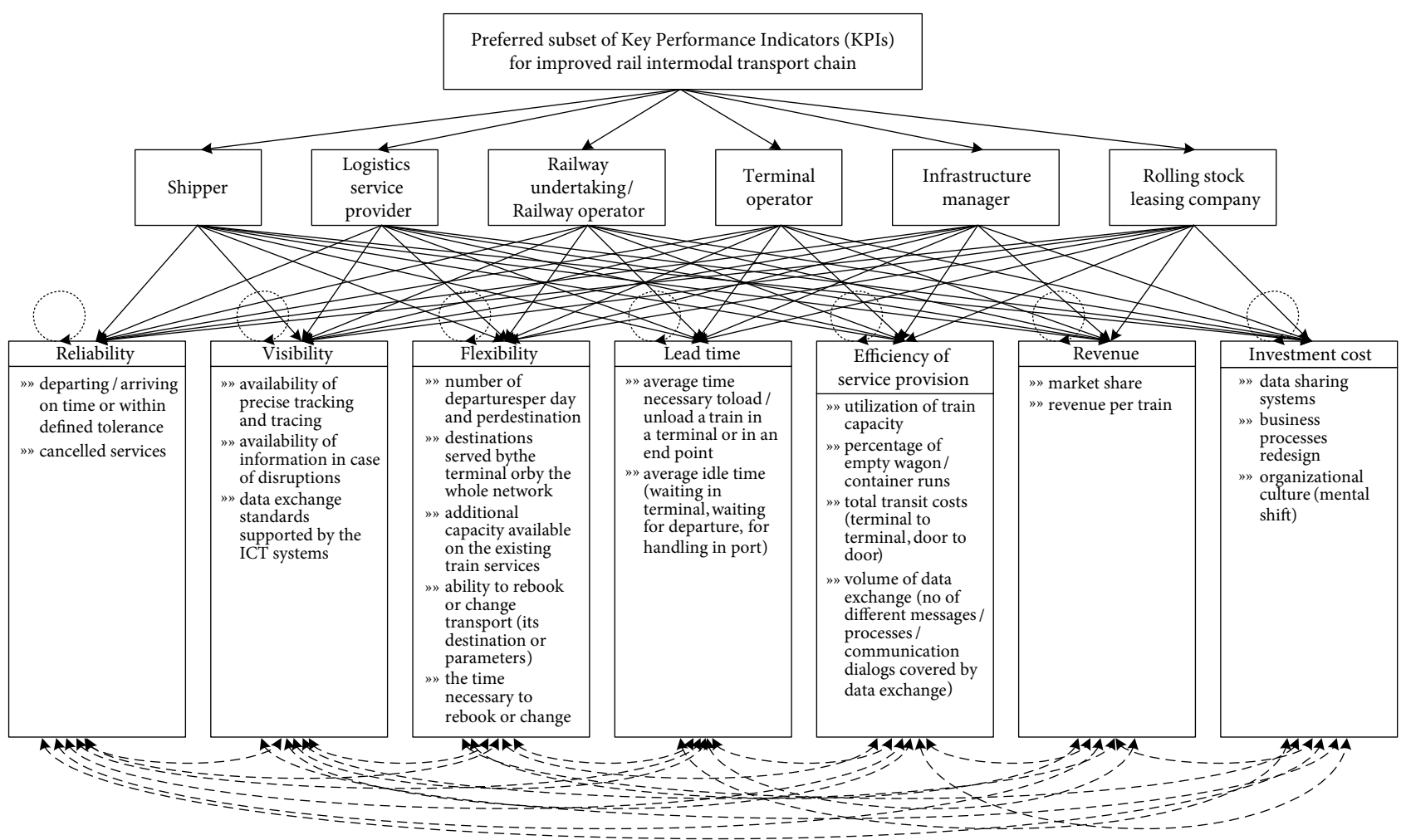

Figure 3. Network structure for prioritizing the set of values associated with rail-enabled information sharing platform 
These attributes are usually interdependent and their weights are hard to obtain. The DEMATEL is used to obtain more accurate weights, whereas ANP is aimed to obtain the interactions between dimensions and attributes in the network. In cases when there are interactions between the attributes in different levels of the hierarchy, then AHP cannot be used because of its one-way direction of hierarchy.

ANP as a more general form of AHP structures the problem as a network where all elements are nodes on the network. In that sense, it allows for feedback connections and loops within and between nodes to illustrate interdependence (Saaty, Vargas 2006). ANP represents the most preferred Multi-Criteria Analysis (MCA) method for modelling dependence and feedback with more than a thousand papers recorded in SCOPUS database since 2005 (Gölcük, Baykasoğlu 2016). Kheybari et al. (2020) provides an extensive overview of ANP-based publications. In ANP applications, it is assumed that the network structure of the problem is known a priori, but in reality, the decision maker cannot form the problem structure easily. The second issue in ANP models is that they quantify the influence between criteria based on pairwise comparisons in which interdependences are treated as reciprocal values, which also does not reflect real situation.

In order to avoid these shortcomings, DEMATEL (Fontela, Gabus 1976), as one of the most popular causal dependency models can be combined with ANP. Hybrid DEMATEL-ANP method (DANP) modifies the original ANP so as to reduce its inherent issues. Based on the NRM the influential relationships are obtained. In contrast to ANP, DANP modifies the pairwise comparisons and forms a comprehensive unweighted supermatrix by building direct influence matrix where pairwise comparisons are not only conducted within clusters but for the whole system according to the structure of the problem. The total relation matrices among the clusters are then used to weight appropriate portions of the supermatrix in order to get the weighted supermatrix. The weighted supermatrix is raised to limiting powers to obtain final priorities (Gölcük, Baykasoğlu 2016; Dehdasht et al. 2017; Tafreshi et al. 2016).

Our strategy is to utilize the described hybrid DEMATEL-ANP approach for determining the level of importance of the set of dimensions / attributes for innovative value proposition in rail intermodal transport. Since the different stakeholders have different preferences the methodology includes an independent analysis for each of them. In the first step, the DEMATEL is used to assess the level of interdependencies between dimensions / attributes and to construct the network relation matrix. In the second step, the relative weights of attributes are calculated based on the combination of ANP and DEMATEL. The last step includes combination of different perspectives and deriving the final weights taking into account relative priority of each of the stakeholders involved. A general overview of the evaluation framework is given in Figure 4. The details of integrated DEMATEL and ANP approach for evaluation of the innovative value proposition for the intermodal rail freight transport are presented in Appendix.

\section{Analysis and discussion of results}

\subsection{Assessment of the innovative value proposition from the aspect of shippers}

The most important attributes that shippers identify with the proposed innovative value proposition are reliability, visibility, flexibility, lead time and investment cost (Figure 5). Deviations between expected and actual time of arrival on loading and unloading location are of crucial importance for shippers. Reliability is particularly important for shippers of fast-moving consumer goods and chemical products. Improving supply chain visibility provides many benefits for shippers - improving on-time delivery, reducing operating costs as well as increasing customer satisfaction. Improved data exchange within the transport chain provides better management and enables flexibility on transhipment points.

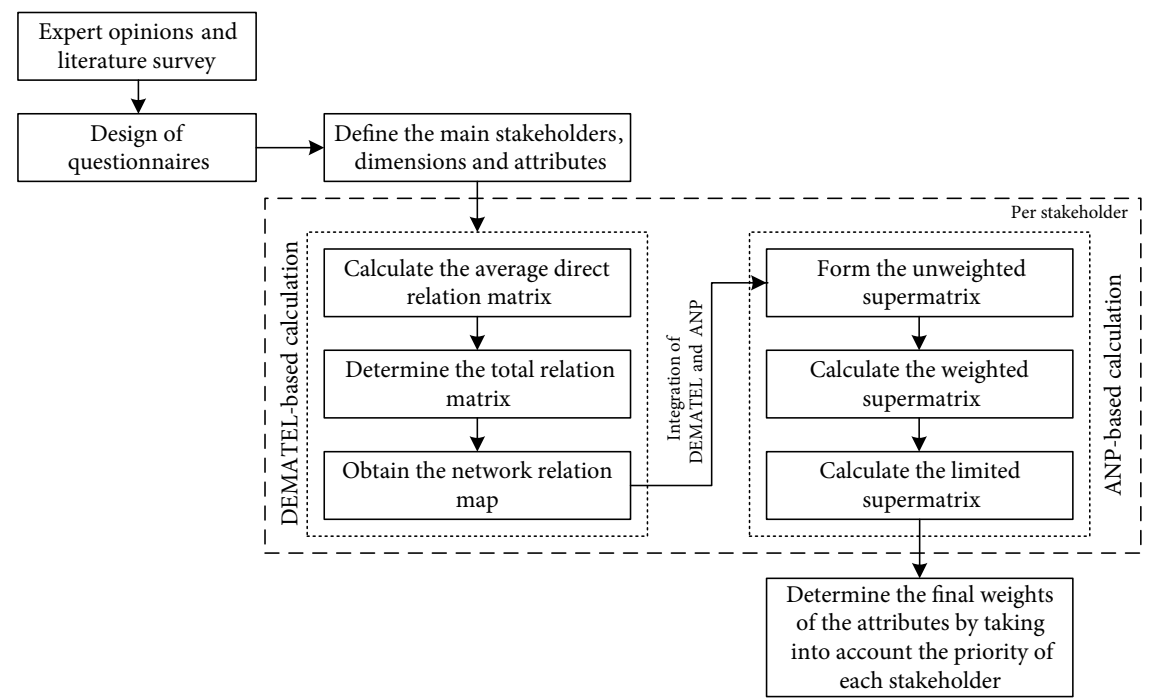

Figure 4. A general overview of the evaluation framework 
New value proposition supports reducing of lead time and lead time variance. Reduced and stable lead times represent very important factors of customer satisfaction and have a direct relationship with the level of inventories at different nodes in the supply chain. The last dimension what shippers identify with innovative value proposition is investment cost that includes the indirect costs of outsourcing the information sharing capabilities.

Influence relationship map for dimensions and criteria that reflect shippers' perspective is presented on Figure 6. Dimensions / criteria that have positive values of $d_{i}-r_{i}$ have significant impact on other dimensions / criteria.

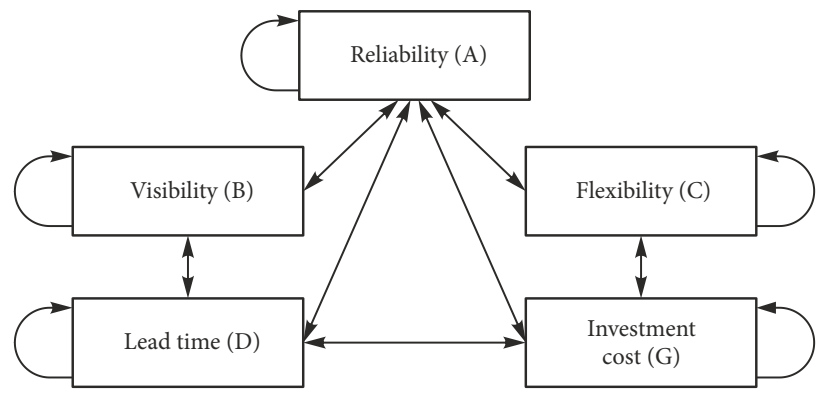

Figure 5. Relationship between dimensions preferred by shippers
These dimensions/criteria are known as net causers. On the other hand, net receivers are dimensions / criteria that have negative value of $d_{i}-r_{i}$ and they are highly dependent on other dimension/criteria. Degree of relationship between dimensions / criteria is determined by value of $d_{i}+r_{i}$. Higher $d_{i}+r_{i}$ value reflects stronger relationship of some dimension / criteria with other dimensions / criteria.

Visibility (B) dimension has the highest $d_{i}-r_{i}$ value, which implies that this dimension has a strong unidirectional impact on other dimensions. The level of visibility (B) of transport flows enables proactive planning of transport processes through higher level of flexibility (C), reliability (A) and shorter lead times (D). Relationship between visibility (B) and investment cost $(\mathrm{G})$ dimensions is obvious since the demand for visibility (B) is directly related to necessary investments in data sharing systems and improvement of business processes. Therefore, visibility (B) dimension should be considered as a priority for improvement from shippers' perspective. Visibility (B) has positive impact on lead time (D) also. Flexibility (C) has a positive impact on reliability (A), lead time (D) and investment cost $(G)$. Investment cost $(G)$ has a positive impact on flexibility (C), reliability (A) and lead time (D).

If we consider significance of influences, we may notice that reliability $(\mathrm{A})$ has the strongest influence $(8.594$

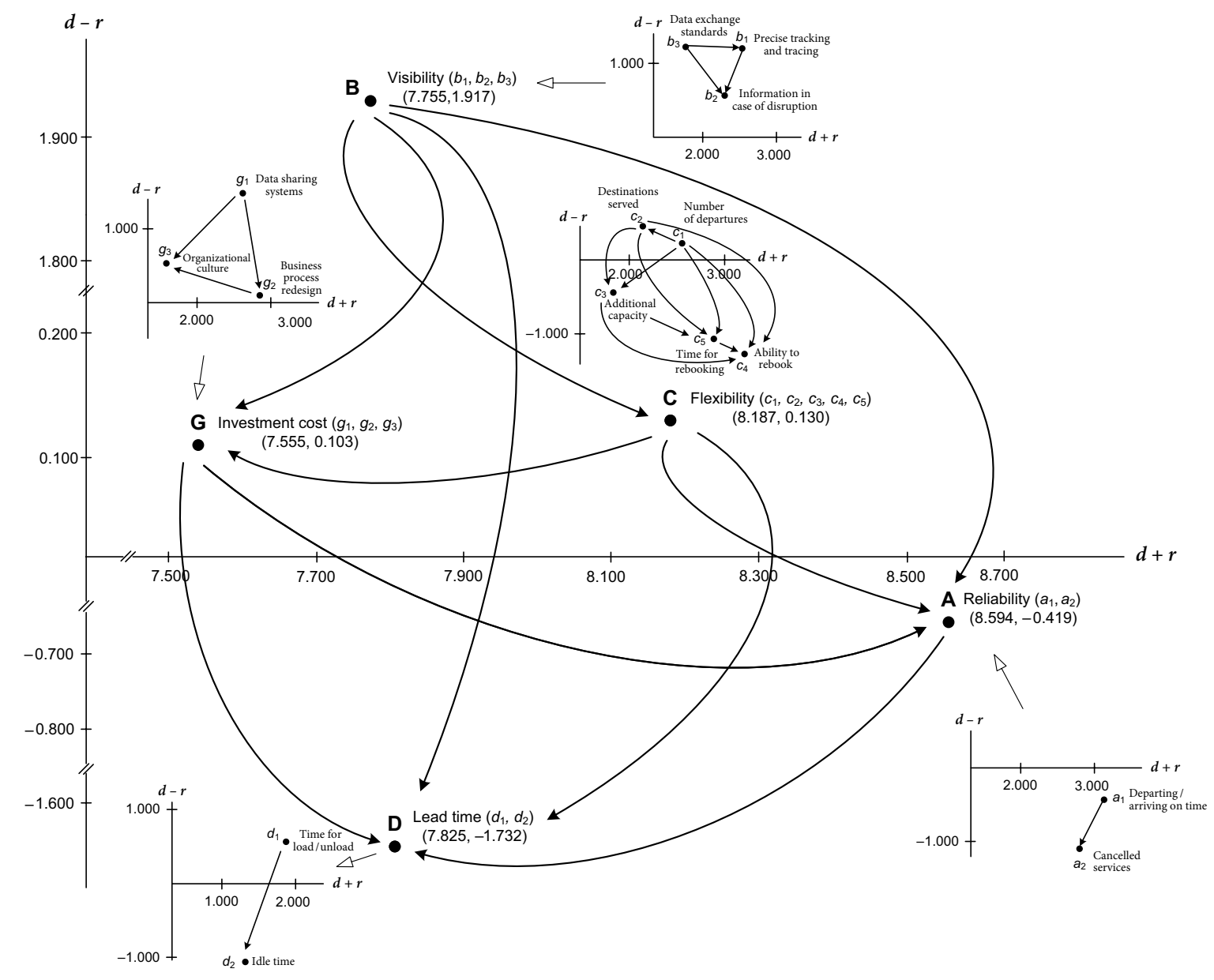

Figure 6. Causal diagram of total relationships: shippers' perspective 
in total sum $d_{A}+r_{A}$ ). Therefore, reliability (A) represents the most influencing factor, whereas investment cost $(G)$ impacts on other factor the least (7.555 in total sum $d_{G}+$ $\left.r_{G}\right)$. From shippers' perspective absence of uncertainty of transportation service strongly affects shippers orientation towards a particular transport mode. Values $d_{i}-r_{i}$ for reliability (A) and lead time (D) are negative, which implies that those dimensions are under the influence of other criteria. Similarly, if we consider the visibility (B) dimension we may notice that precise tracking and tracing $b_{1}$ is the first in terms of index of strength of influence given and received, information in case of disruption $d_{2}$ is next and data exchange standards $b_{3}$ are on third place. In addition, data exchange standards have the highest $d_{i}-r_{i}$ value, which means that they affect other factors in visibility (B). In case of dimension reliability (A) departing / arriving on-time $a_{1}$ has the highest significance and impact on the level of cancelled services $a_{2}$. In the flexibility (C) panel, ability to rebook $c_{4}$ has the highest influence given and received, time interval needed for rebooking $c_{5}$ is next, followed by the number of departures $c_{1}$, number of destinations served $c_{2}$ and additional capacity $c_{3}$. In the lead time (D) panel time for loading / unloading $d_{1}$ is the first in terms of the index of strength of influence given and received, which also has the impact on idle time $d_{2}$ due to high $d_{i}-r_{i}$ value. Finally, in investment cost $(\mathrm{G})$ panel business process redesign $g_{2}$ has the highest index of strength given and received followed by data sharing systems $g_{1}$ and organization culture $g_{3}$.

If we consider the extent of the impact of each criterion, departing / arriving on-time or within defined tolerance $a_{1}$ is the most important consideration $\left(d_{a 1}+r_{a 1}=3.117\right)$ whereas organizational culture $g_{3}$ is the criteria with least impact on the other criteria $\left(d_{g 3}+r_{g 3}=0.972\right) \cdot d_{g 1}-r_{g 1}$ for data sharing systems $g_{1}$ shows that this criterion has the greatest direct impact on others $\left(d_{g 1}-r_{g 1}=1.493\right)$ in total difference, whereas ability to rebook or change transport $c_{4}$ is the criterion, which is most easily influenced by other criteria $\left(d_{c 4}-r_{c 4}=-1.263\right)$.

Influential weights for all criteria, which are preferred by the shippers are obtained by following the DANP approach (Appendix). Weighted supermatrix $W_{W}$ is obtained by integration unweighted supermatrix into the total influence matrix $T_{D}$. Limited supermatrix whose vectors represent relative weights of the criteria is calculated by raising the weighted supermatrix $W_{W}$ to a sufficient power $k$, until the convergence of the supermatrix.

Table 5 contains relative importance of dimensions and criteria, as well as their rank. Among the 15 criteria preferred by the shippers, the most important are related to reliability (A) dimension: number of cancelled services $a_{2}$ with a weight of 0.164 , followed by the departing / arriving on-time $a_{1}(0.149)$. The 3 rd to 10 th criteria in order of importance from greatest to least are: average idle time $d_{2}(0.142)$, ability to rebook or change transport $c_{4}(0.086)$, organizational culture $g_{3}(0.072)$, business process redesign $g_{2}(0.069)$, time necessary to rebook $c_{5}(0.064)$, time necessary to load / unload $d_{1}(0.064)$, number of departures per day $c_{1}(0.048)$ and additional capacity available on existing train services $c_{3}(0.043)$. Among the top ten criteria, there are two from reliability (A) dimension, two from lead time (D) dimension, two from investment cost (G) dimension and four from flexibility (C) dimension. Criteria that belong to visibility (B) is less important.

Table 5. Influential weights of DEMATEL-ANP for each criterion obtained by $\lim _{n \rightarrow \infty}\left(W^{\alpha}\right)^{n}:$ shippers' perspective

\begin{tabular}{|c|c|c|c|c|}
\hline Dimension & $\begin{array}{c}\text { Relative weight } \\
\text { of dimension }\end{array}$ & Criteria & $\begin{array}{l}\text { Relative weight of } \\
\text { criteria }\end{array}$ & Rank \\
\hline \multirow{2}{*}{ Reliability (A) } & \multirow{2}{*}{0.313} & departing / arriving on-time or within defined tolerance $a_{1}$ & 0.149 & 2 \\
\hline & & cancelled services $a_{2}$ & 0.164 & 1 \\
\hline \multirow{3}{*}{ Visibility (B) } & \multirow{3}{*}{0.046} & availability of precise tracking and tracing $b_{1}$ & 0.019 & 13 \\
\hline & & availability of information in case of disruptions $b_{2}$ & 0.018 & 14 \\
\hline & & data exchange standards supported by the ICT systems $b_{3}$ & 0.009 & 15 \\
\hline \multirow{5}{*}{ Flexibility (C) } & \multirow{5}{*}{0.270} & number of departures per day and per destination $c_{1}$ & 0.048 & 9 \\
\hline & & destinations served by the terminal or by the whole network $c_{2}$ & 0.029 & 11 \\
\hline & & additional capacity available on the existing train services $c_{3}$ & 0.043 & 10 \\
\hline & & ability to rebook or change transport (its destination or parameters) $c_{4}$ & 0.086 & 4 \\
\hline & & the time necessary to rebook or change $c_{5}$ & 0.064 & 7 \\
\hline \multirow{2}{*}{$\begin{array}{l}\text { Lead time } \\
\text { (D) }\end{array}$} & \multirow{2}{*}{0.206} & $\begin{array}{l}\text { average time necessary to load / unload a train in a terminal or in an } \\
\text { end point } d_{1}\end{array}$ & 0.064 & 8 \\
\hline & & $\begin{array}{l}\text { average idle time (waiting in terminal, waiting for departure, for } \\
\text { handling in port) } d_{2}\end{array}$ & 0.142 & 3 \\
\hline \multirow{3}{*}{$\begin{array}{l}\text { Investment } \\
\text { cost }(G)\end{array}$} & \multirow{3}{*}{0.161} & data sharing systems $g_{1}$ & 0.020 & 12 \\
\hline & & business processes redesign $g_{2}$ & 0.069 & 6 \\
\hline & & organizational culture (mental shift) $g_{3}$ & 0.072 & 5 \\
\hline
\end{tabular}




\subsection{Assessment of the innovative value proposition from the aspect of LSPs}

The most preferred aspects of innovative value proposition for LSPs are flexibility, visibility, lead time and investment cost (Figure 7). Logistics operations that are managed without contingencies for the disruptions offer poor services and result in higher costs (Ishfaq 2012).

Therefore, it is of crucial importance for LSPs to consider potential disruptions during the planning phase in order to minimize the risk of poor performance of logistics operations in case disruptions along the transport chain suddenly appear. Lead time as the total time that elapses between an order's placement and its receipt has serious effects on the coordination between actors in transport

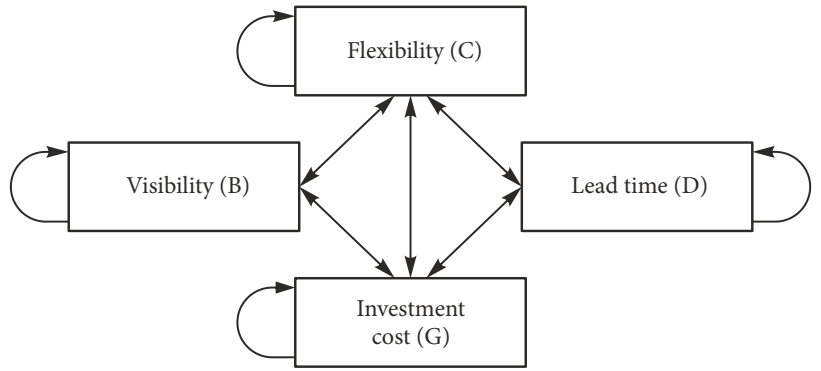

Figure 7. Relationship between dimensions preferred by LSPs

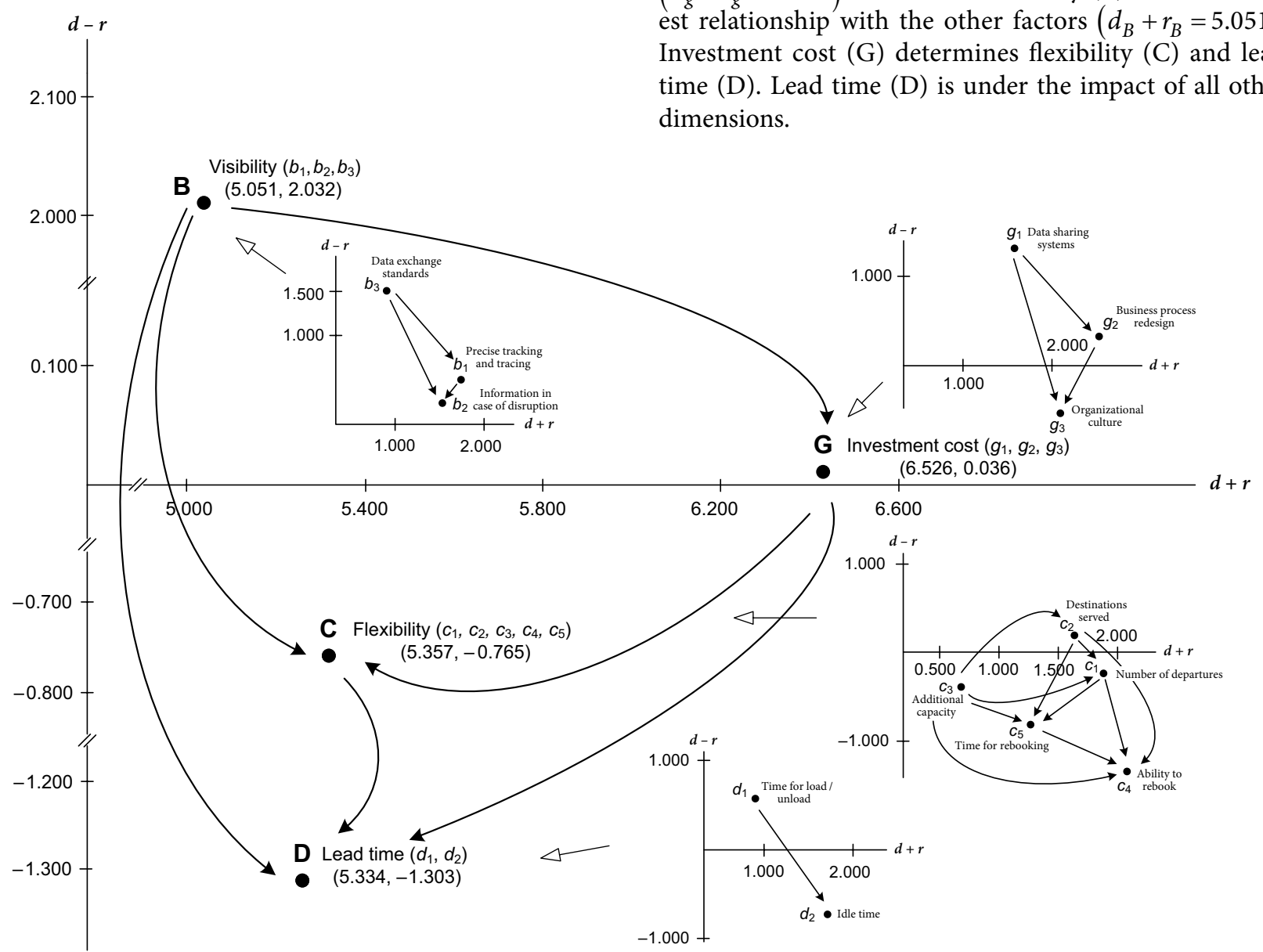

chain and it can be viewed as a coordination enabler. In case of food and beverages manufacturers outsourcing of logistics activities to a specific LSP on the rate of inventory replenishment (Wambua et al. 2017).

LSPs operate in a very challenging environment and faster and reliable information sharing in rail intermodal transport chain contributes to decreasing of their costs and increasing of their competitiveness. Visibility enables acting of LSPs on operational costs and supply chain finances and developing of proactive strategies with customers. Investment cost reflected in a participation fee or IT system integration or even building the platform (if LSP acts as a transport chain orchestrator) represents a potential barrier to the proposed new value proposal.

Figure 8 illustrates relationships between dimensions and criteria identified by LSPs. Like in case of shippers, visibility (B) dimension has the highest positive values of $d_{i}-r_{i}\left(d_{B}-r_{B}=2.032\right)$ and thus greatly influence on other dimensions. Visibility (B) enables higher level of flexibility (C) and shorter lead time (D). Its impact on investment cost $(G)$ is reflected in investments in data sharing as well as in redesign of business process and organization culture. On the other side, flexibility (C) and lead time (D) are greatly influenced by other dimensions since they have negative values of $d_{i}-r_{i},-0.765$ and -1.303 , respectively. For LSPs, investment cost $(\mathrm{G})$ has the strongest influence $\left(d_{g}+r_{g}=6.526\right)$ whereas the visibility (B) has the weakest relationship with the other factors $\left(d_{B}+r_{B}=5.051\right)$. Investment cost $(\mathrm{G})$ determines flexibility $(\mathrm{C})$ and lead time (D). Lead time (D) is under the impact of all other dimensions.

Figure 8. Causal diagram of total relationships: LSPs' perspective 
Within investment cost (G), according to the LSPs, data sharing systems $g_{1}$ give their influence to the other criteria at most $\left(d_{g 1}-r_{g 1}=1.110\right)$ whereas business process redesign $g_{2}$ is viewed as the criteria with strongest relationship with other criteria $\left(d_{g 1}+r_{g 1}=2.303\right)$. If we consider flexibility (C) dimension in case of LSPs, only criteria destinations served $c_{2}$ has positive $d_{i}-r_{i}$. Ability to rebook $c_{4}$ has the highest $r_{i}+d_{i}$ and it represents the most influencing factor. Criteria that belong to visibility (B) dimension have the same mutual relationship like the same considered from shippers perspective. Within lead time (D) dimension, average idle time $d_{2}$ has the highest influence for LSPs $\left(r_{d 2}+d_{d 2}=1.516\right)$. Lead time (D) depends on flexibility $(\mathrm{C})$.

Relative importance of dimensions and criteria as well as their rank are presented in Table 6. Among the 13 criteria selected by LSPs, the most important are related to lead time (D) dimension: average idle time with a weight $d_{2}$ of 0.247 , followed by ability to rebook or change transport $c_{4}(0.147)$ and organizational culture $g_{3}(0.126)$. The rest of criteria are ranked in following order: business process redesign $g_{2}(0.115)$, time necessary to rebook or change $c_{5}(0.069)$, destinations served by the terminal $c_{2}(0.055)$, availability of precise tracking and tracing $b_{1}$ $(0.033)$, availability of information in case of disruptions $b_{2}(0.032)$, data sharing systems $g_{1}(0.029)$, additional capacity available on existing train services $c_{3}(0.021)$, data exchange standards $b_{3}(0.018)$ and average time necessary to load / unload a train $d_{1}(0.016)$.

\subsection{Assessment of the innovative value proposition from the aspect of terminal operators}

Terminal operators identify four main aspects of innovative value proposition, reliability, efficiency of service provision, visibility and investment cost (Figure 9). Reliability represents a critical performance condition for terminal

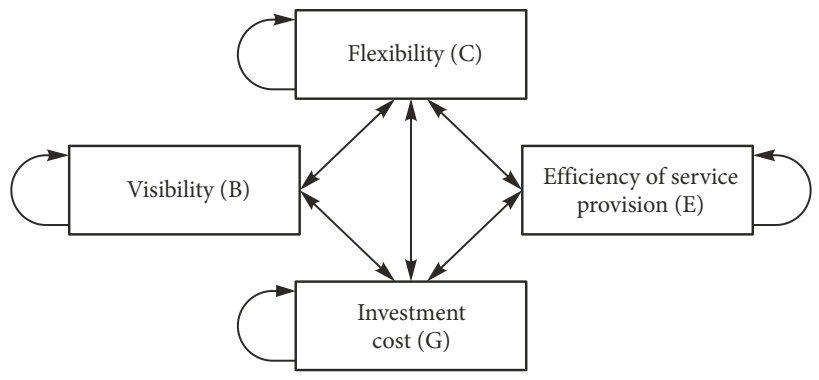

Figure 9. Relationship between dimensions preferred by Terminal Operators

operators. It enables reducing of the impedance of transhipment operations and contributes to increased efficiency and effectiveness of terminal's processes. Efficiency of service provision enabled by innovative value proposition contributes to higher utilization and productivity of terminal facilities. Visibility enables improved coordination, proactive decision-making and more synchronized intermodal logistics process. This allows terminals to achieve a decrease in operating times for cargo handling, including improvements in waiting times for cargo arriving and departing. Terminal operators also identify the cost of investing in new value proposition through integration of systems or paying a participation fee. However, they expect that this cost will be outbalanced by increment in profit expected from higher volume of handling and efficiency of terminal operations.

Figure 10 represents impact relation map for main dimensions and criteria identified by terminal operators. It can be seen in the middle panel (dimension) that investment cost $(G)$ is the first with index of strength of influence given and received (3.709 in total sum $d_{G}+r_{G}$ ). Visibility (B) is next $\left(d_{B}+r_{B}=3.085\right)$, then reliability (A) and efficiency of service provision (E) follow with index of strength of influence 2.714 and 2.692, respectively.

Table 6. Influential weights of DEMATEL-ANP for each criterion obtained by $\lim _{n \rightarrow \infty}\left(W^{\alpha}\right)^{n}:$ LSPs' perspective

\begin{tabular}{|c|c|c|c|c|}
\hline Dimension & $\begin{array}{c}\text { Relative weight } \\
\text { of dimension }\end{array}$ & Criteria & $\begin{array}{c}\text { Relative weight } \\
\text { of criteria }\end{array}$ & Rank \\
\hline \multirow{3}{*}{ Visibility (B) } & \multirow{3}{*}{0.083} & availability of precise tracking and tracing $b_{1}$ & 0.033 & 8 \\
\hline & & availability of information in case of disruptions $b_{2}$ & 0.032 & 9 \\
\hline & & data exchange standards supported by the ICT systems $b_{3}$ & 0.018 & 12 \\
\hline \multirow{5}{*}{ Flexibility (C) } & \multirow{5}{*}{0.378} & number of departures per day and per destination $c_{1}$ & 0.086 & 5 \\
\hline & & destinations served by the terminal or by the whole network $c_{2}$ & 0.055 & 7 \\
\hline & & additional capacity available on the existing train services $c_{3}$ & 0.021 & 11 \\
\hline & & ability to rebook or change transport (its destination or parameters) $c_{4}$ & 0.147 & 2 \\
\hline & & the time necessary to rebook or change $c_{5}$ & 0.069 & 6 \\
\hline \multirow{2}{*}{ Lead time $(\mathrm{D})$} & \multirow{2}{*}{0.263} & $\begin{array}{l}\text { average time necessary to load / unload a train in a terminal or in an } \\
\text { end point } d_{1}\end{array}$ & 0.016 & 13 \\
\hline & & $\begin{array}{l}\text { average idle time (waiting in terminal, waiting for departure, for } \\
\text { handling in port) } d_{2}\end{array}$ & 0.247 & 1 \\
\hline \multirow{3}{*}{$\begin{array}{l}\text { Investment } \\
\text { cost }(G)\end{array}$} & \multirow{3}{*}{0.270} & data sharing systems $g_{1}$ & 0.029 & 10 \\
\hline & & business processes redesign $g_{2}$ & 0.115 & 4 \\
\hline & & organizational culture (mental shift) $g_{3}$ & 0.126 & 3 \\
\hline
\end{tabular}




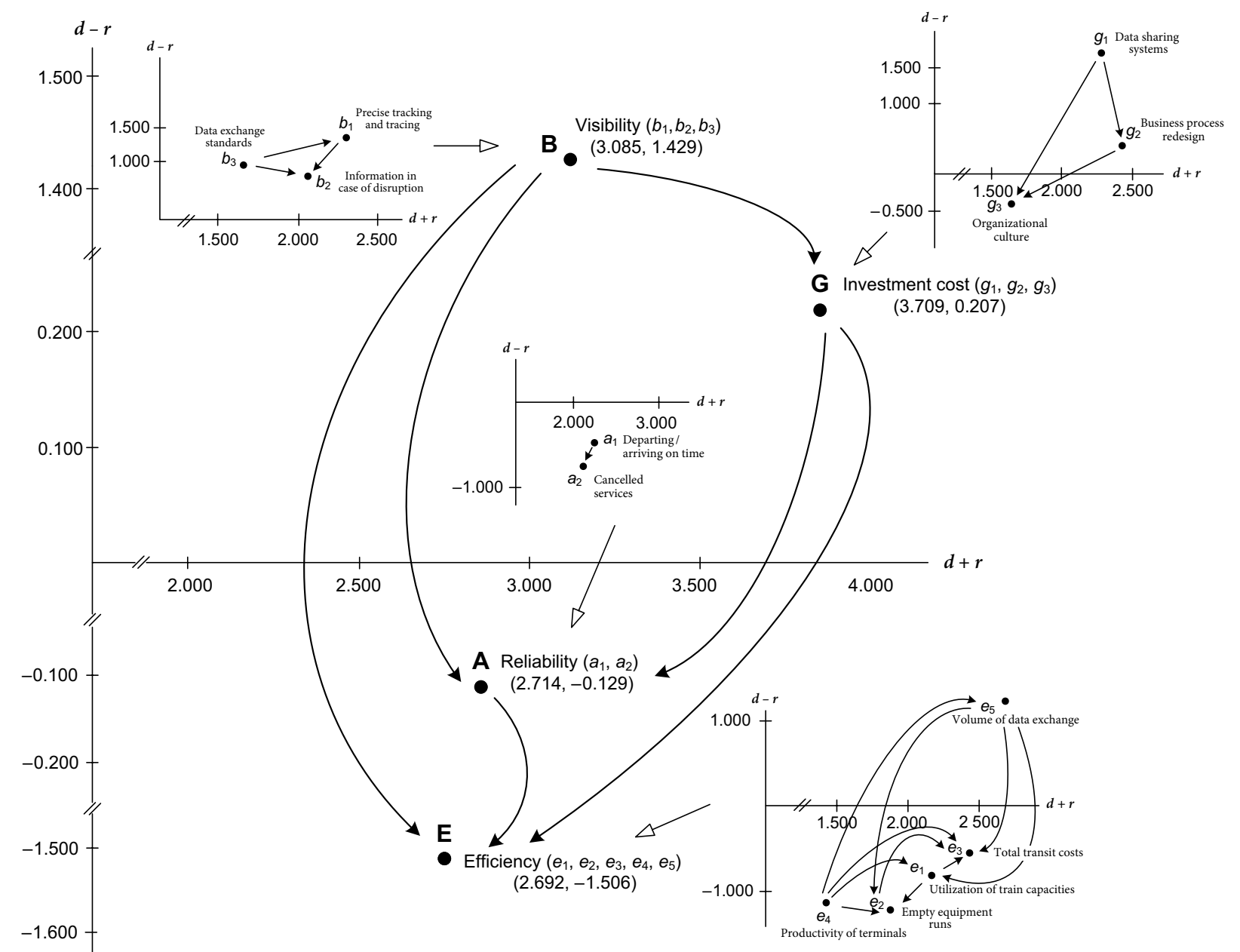

Figure 10. Causal diagram of total relationships: terminal operators' perspective

Values of $d_{i}-r_{i}$ for visibility (B) and investment cost (G) are positive meaning that they affect other criteria. Values of $d_{i}-r_{i}$ for reliability (A) and efficiency of service provision (E) dimensions are negative, which means that these criteria are influenced by other criteria. In the investment cost (G) panel it can be seen that business process redesign $g_{2}$ is the first in terms of the index of strength given and received $\left(d_{g 2}+r_{g 2}=2.469\right)$. Data sharing system $g_{1}$ affect other criteria in the dimension $\left(d_{g 1}+r_{g 1}=2.379\right)$. In the visibility (B) panel precise tracking and tracing $b_{1}$ has the strongest influence $\left(d_{b 1}+r_{b 1}=2.284\right)$ and it also affects the other criteria at most $\left(d_{b 1}-r_{b 1}=1.386\right)$. In reliability (A) panel departing / arriving on-time $a_{1}$ affects the number of cancelled services $a_{2}$. Within efficiency of service provision (E) volume of data exchange $e_{5}$ is the first in the index of strength of influence given and received $\left(d_{e 5}+r_{e 5}=2.758\right)$ and it has significantly positive $d_{i}-r_{i}$ value. All other criteria have negative $d_{i}-r_{i}$ value.

According to Table 7, criteria that belong to reliability (A) dimension have the highest importance: cancelled services $a_{2}$ with relative weight $(0.183)$ followed by criteria departing / arriving on-time or within defined tolerance $a_{1}(0.165)$. Efficiency of service provision (E) is the dimension with highest relative weight $(0.376)$ with highly ranked three of five criteria: reduced total transit costs $e_{3}$ $(0.095)$ as third, reduced \% of empty wagon / container runs $e_{2}(0.092)$ as fourth and increased utilization of train capacity $e_{1}(0.089)$ as fifth criteria in the list. The 6th to 13 th criteria in order of importance from greatest to least are: business process redesign $g_{1}(0.086)$, organizational culture $g_{3}(0.082)$, productivity of terminal's facilities $e_{4}$ (0.074), availability of information in case of disruptions $b_{2}(0.032)$, availability of precise tracking and tracing $b_{1}$ (0.027), data exchange standards $b_{3}(0.026)$, volume of data exchange $e_{5}(0.026)$, data sharing systems $g_{1}(0.022)$ and data exchange standards supported by the ICT systems $b_{3}(0.022)$.

\subsection{Assessment of the innovative value proposition from the aspect of railway operators}

Railway operators expect better results regarding reliability, revenue and efficiency of service provision (Figure 11). The reliability represents one of the main problems of rail freight transport and significantly affects the prospective customers in determination of transport mode. New value proposition removes barriers in information sharing between the railway and non-railway related stakeholders and enables more intensive integration of railways in the 
Table 7. Influential weights of DEMATEL-ANP for each criterion obtained by $\lim _{n \rightarrow \infty}\left(W^{\alpha}\right)^{n}:$ terminal operators' perspective

\begin{tabular}{|c|c|c|c|c|}
\hline Dimension & $\begin{array}{l}\text { Relative weight } \\
\text { of dimension }\end{array}$ & Criteria & $\begin{array}{c}\text { Relative weight } \\
\text { of criteria }\end{array}$ & Rank \\
\hline \multirow{2}{*}{ Reliability (A) } & \multirow{2}{*}{0.348} & departing / arriving on-time or within defined tolerance $a_{1}$ & 0.165 & 2 \\
\hline & & cancelled services $a_{2}$ & 0.183 & 1 \\
\hline \multirow{3}{*}{ Visibility (B) } & \multirow{3}{*}{0.081} & availability of precise tracking and tracing $b_{1}$ & 0.027 & 10 \\
\hline & & availability of information in case of disruptions $b_{2}$ & 0.032 & 9 \\
\hline & & data exchange standards supported by the ICT systems $b_{3}$ & 0.022 & 13 \\
\hline \multirow{5}{*}{$\begin{array}{l}\text { Efficiency of service } \\
\text { provision (E) }\end{array}$} & \multirow{5}{*}{0.376} & utilization of train capacity $e_{1}$ & 0.089 & 5 \\
\hline & & percentage of empty wagon / container runs $e_{2}$ & 0.092 & 4 \\
\hline & & total transit costs (terminal to terminal, door to door) $e_{3}$ & 0.095 & 3 \\
\hline & & productivity of terminal facilities $e_{4}$ & 0.074 & 8 \\
\hline & & volume of data exchange $e_{5}$ & 0.026 & 11 \\
\hline \multirow{3}{*}{ Investment cost $(\mathrm{G})$} & \multirow{3}{*}{0.190} & data sharing systems $g_{1}$ & 0.022 & 12 \\
\hline & & business processes redesign $g_{2}$ & 0.086 & 6 \\
\hline & & organisational culture (mental shift) $g_{3}$ & 0.082 & 7 \\
\hline
\end{tabular}

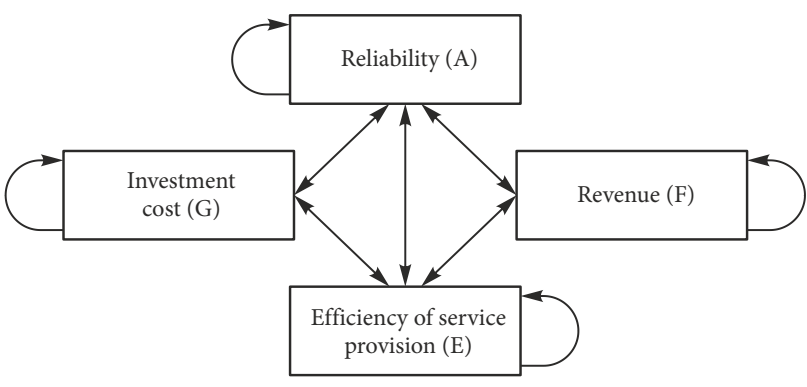

Figure 11. Relationship between dimensions preferred by railway undertakings / railway operators

transport chain. This will contribute to the improved competitiveness of railway operators, higher level of efficiency of service provision and increased revenue. Cost related to investment in new value proposition represents very important attribute for railway operators. This cost includes integration of "closed" railway related information systems into a rail-enabled information sharing platform, business processes redesign in order to enable transition from traditional to a more customer oriented operating policy and a mental shift toward the more supply chainoriented mind set.

Investment cost $(\mathrm{G})$ with highest value $r_{i}+d_{i}(3.273)$ has the strongest influence for acceptance of new value proposition from railway operators (Figure 12). Investment cost $(G)$ impacts on reliability (A), efficiency of service provision (E) and revenue (F). Reliability (A) has the highest $d_{i}-r_{i}$ value with the strongest impact on other dimensions (0.994). Within investment cost (G) dimension, railway operators identify process redesign as the most important in terms of index of strength of influence given and received. This is related to very complex organizational structure and processes. In efficiency of service provision (E) dimension utilization of train capacities $e_{1}$ has the highest value $\left(r_{e 1}+d_{e 1}=7.038\right)$ with a strong influence on other efficiency related criteria: empty container runs $e_{2}$, total transit costs $e_{3}$ and productivity of terminal facilities $e_{4}$. Railway operators consider cancelled services $a_{2}$ as the most important in reliability (A) dimension $\left(r_{a 2}+d_{a 2}=6.330\right)$. The main aspect of revenue (F) dimension is increased market share $f_{1}$, which determines the level of revenue per $\operatorname{train} f_{2}$.

According to the results in Table 8, revenue (F) dimension has the largest weight $(0.319)$ followed by efficiency of service provision (E) (0.260), reliability (A) (0.253) and investment cost $(\mathrm{G})$ (0.163). In terms of criteria, revenue per train $f_{2}(0.161)$, market share $f_{1}(0.158)$ and cancelled services $a_{2}(0.127)$ are the three most significant. On the other hand, total transit costs $e_{3}(0.047)$, volume of data exchange $e_{5}(0.039)$ and data sharing systems $g_{1}(0.035)$ are the least important criteria for railway operators.

\subsection{Assessment of the innovative value proposition from the aspect of infrastructure manager}

In case of infrastructure managers, higher level of revenue for rail operators will indirectly bring financial benefits to the infrastructure manager (Figure 13).

Reliability represents one of the key prerequisites for developing and optimizing the rail transport infrastructure. Improved visibility on rail enables infrastructure manager to use the information about physical location and movement of rail vehicles for more efficient train traffic control on rail network. However, infrastructure managers expect that joining to the collective value proposition would produce the costs related to integration of existing "closed" systems with the other stakeholders in the information sharing platform as well as the efforts related to the shift of traditional mind set of railway infrastructure managers to a more supply chain orientated mind set.

As we can see from Figure 14, the largest $d_{i}-r_{i}$ value (0.918) is for visibility (B). Therefore, the visibility (B) will have a strong influence on other dimensions. 


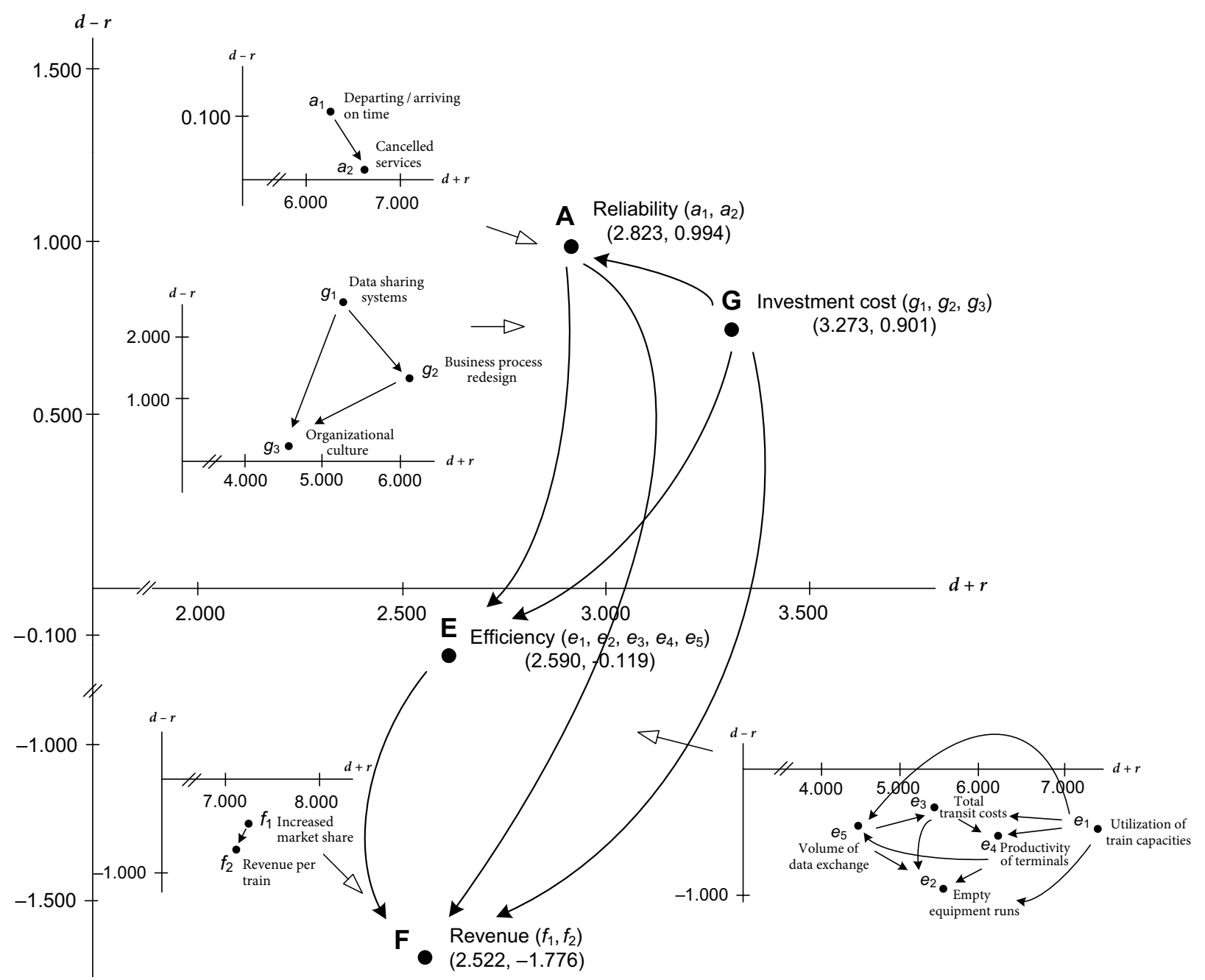

Figure 12. Causal diagram of total relationships: railway operators' perspective

Table 8. Influential weights of DEMATEL-ANP for each criterion obtained by $\lim _{n \rightarrow \infty}\left(W^{\alpha}\right)^{n}:$ railway operators' perspective

\begin{tabular}{|c|c|c|c|c|}
\hline Dimension & $\begin{array}{l}\text { Relative weight } \\
\text { of dimension }\end{array}$ & Criteria & $\begin{array}{l}\text { Relative weight } \\
\text { of criteria }\end{array}$ & Rank \\
\hline \multirow{2}{*}{ Reliability (A) } & \multirow{2}{*}{0.253} & departing / arriving on-time or within defined tolerance $a_{1}$ & 0.126 & 4 \\
\hline & & cancelled services $a_{2}$ & 0.127 & 3 \\
\hline \multirow{5}{*}{$\begin{array}{l}\text { Efficiency } \\
\text { of service } \\
\text { provision (E) }\end{array}$} & \multirow{5}{*}{0.260} & increased utilization of train capacity $e_{1}$ & 0.063 & 6 \\
\hline & & reduced \% of empty wagon / container runs $e_{2}$ & 0.054 & 9 \\
\hline & & reduced total transit costs (terminal to terminal, door to door) $e_{3}$ & 0.047 & 10 \\
\hline & & productivity of terminal facilities $e_{4}$ & 0.057 & 8 \\
\hline & & volume of data exchange $e_{5}$ & 0.039 & 11 \\
\hline \multirow{2}{*}{ Revenue (F) } & \multirow{2}{*}{0.319} & market share $f_{1}$ & 0.158 & 2 \\
\hline & & revenue per train $f_{2}$ & 0.161 & 1 \\
\hline \multirow{3}{*}{$\begin{array}{l}\text { Investment } \\
\text { cost }(G)\end{array}$} & \multirow{3}{*}{0.163} & data sharing systems $g_{1}$ & 0.035 & 12 \\
\hline & & business processes redesign $g_{2}$ & 0.068 & 5 \\
\hline & & organisational culture (mental shift) $g_{3}$ & 0.060 & 7 \\
\hline
\end{tabular}

In addition, investment cost $(\mathrm{G})$ has the maximum value $\left(d_{i}+r_{i}=2.679\right)$, which means that it has the largest total influence degree within dimensions. Reliability (A) and revenue (F) are affected by the investments in data sharing systems $g_{1}$, business process redesign $g_{2}$ and changing of mind set of railway operators. Customer satisfaction and revenue are under the impact of reliability (A). In investment cost $(\mathrm{G})$ dimension, data sharing systems $g_{1}$, influences on business process redesign $g_{2}$ and organizational culture $g_{3}$. This means that the data sharing systems imply the need for redesign of existing business processes and changing the traditional mind set (supply chain orientation). 
In visibility $(B)$ dimension, there is a strong relationship of availability of data exchange standards $b_{3}$ on precise tracking and tracing $b_{1}$ and on availability of information in case of disruption $b_{2}$. In reliability $(\mathrm{A})$ dimension departing / arriving on-time $a_{1}$ impacts on the level of can-

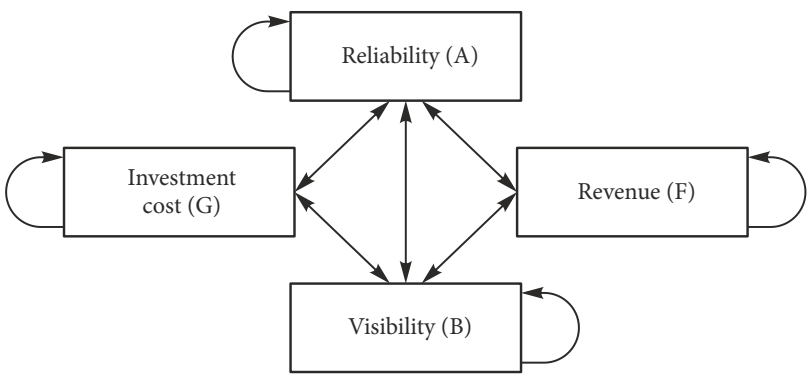

Figure 13. Relationship between dimensions preferred by Infrastructure Manager celled services $a_{2}$. Increased market share $f_{1}$ is the most important criteria in revenue $(\mathrm{F})$ dimension and it has a strong impact on revenue per $\operatorname{train} f_{2}$.

From Table 9, revenue (F) has the largest weight followed by reliability (A), investment cost $(\mathrm{G})$ and visibility (B). In terms of criteria, increased market share $f_{1}(0.177)$, revenue per train $f_{2}(0.176)$ and departing / arriving ontime or within the defined tolerance $a_{1}(0.139)$ are the most important criteria. On the other hand, availability of precise tracking and tracing $b_{1}(0.056)$, data sharing systems $g_{1}(0.052)$ and data exchange standards $b_{3}(0.048)$ are the least important criteria for infrastructure managers.

\subsection{Assessment of the innovative value proposition from the aspect of rolling stock leasing companies}

Rail-enabled information sharing platform enables improved productivity of rail freight assets and supports the implementation of predictive models and tools for rolling

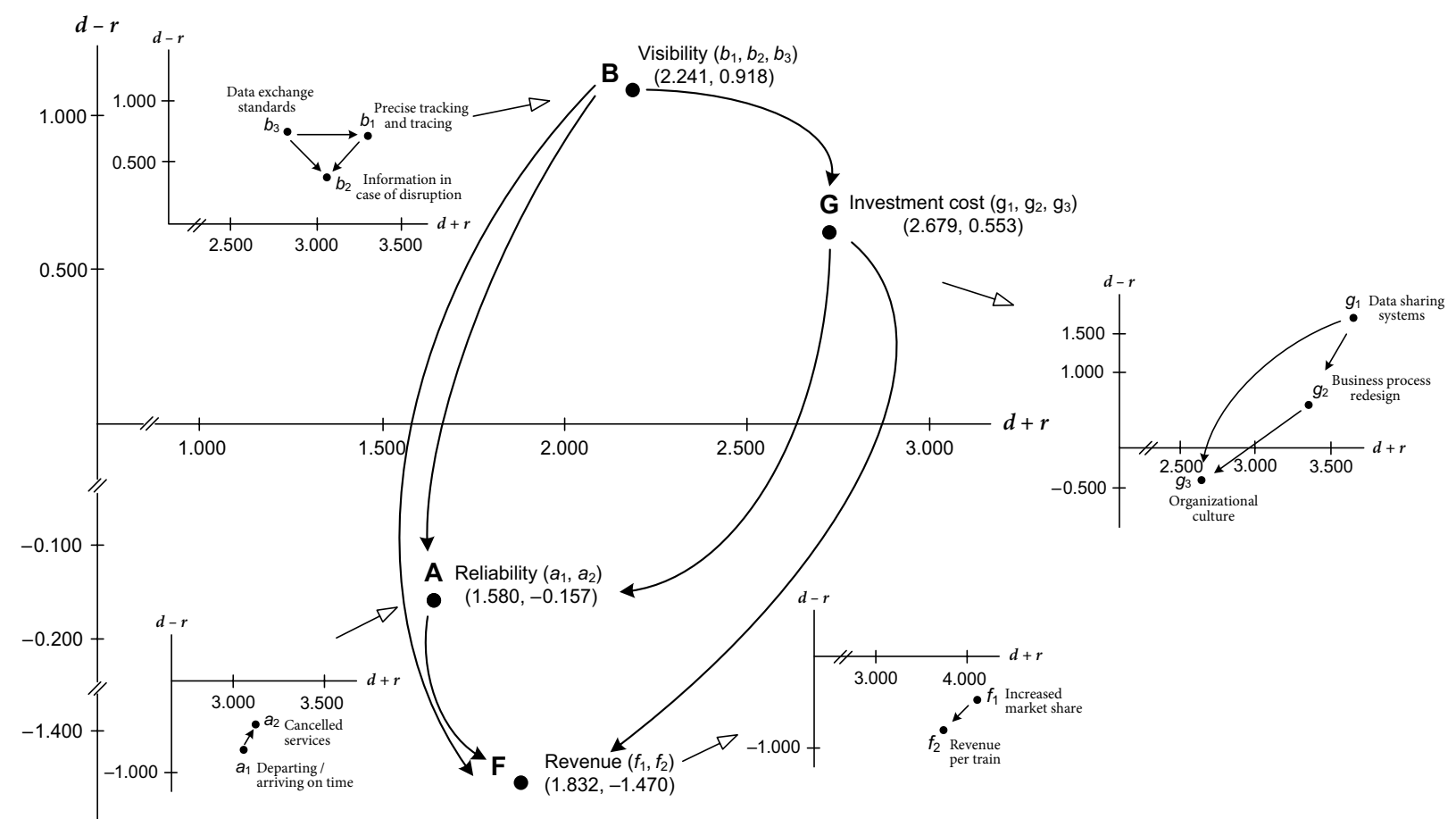

Figure 14. Causal diagram of total relationships: infrastructure manager' perspective

Table 9. Influential weights of DEMATEL-ANP for each criterion obtained by $\lim _{n \rightarrow \infty}\left(W^{\alpha}\right)^{n}$ : infrastructure managers' perspective

\begin{tabular}{|c|c|c|c|c|}
\hline Dimension & $\begin{array}{l}\text { Relative weight } \\
\text { of dimension }\end{array}$ & Criteria & $\begin{array}{l}\text { Relative weight } \\
\text { of criteria }\end{array}$ & Rank \\
\hline \multirow{2}{*}{ Reliability (A) } & \multirow{2}{*}{0.278} & departing / arriving on-time or within defined tolerance $a_{1}$ & 0.139 & 3 \\
\hline & & cancelled services $a_{2}$ & 0.139 & 4 \\
\hline \multirow{3}{*}{ Visibility (B) } & \multirow{3}{*}{0.162} & availability of precise tracking and tracing $b_{1}$ & 0.056 & 8 \\
\hline & & availability of information in case of disruptions $b_{2}$ & 0.058 & 7 \\
\hline & & data exchange standards supported by the ICT systems $b_{3}$ & 0.048 & 10 \\
\hline \multirow{2}{*}{ Revenue (F) } & \multirow{2}{*}{0.353} & increased market share $f_{1}$ & 0.177 & 1 \\
\hline & & revenue per $\operatorname{train} f_{2}$ & 0.176 & 2 \\
\hline \multirow{3}{*}{$\begin{array}{l}\text { Investment } \\
\text { cost }(G)\end{array}$} & \multirow{3}{*}{0.202} & data sharing systems $g_{1}$ & 0.052 & 9 \\
\hline & & business processes redesign $g_{2}$ & 0.074 & 6 \\
\hline & & organisational culture (mental shift) $g_{3}$ & 0.076 & 5 \\
\hline
\end{tabular}




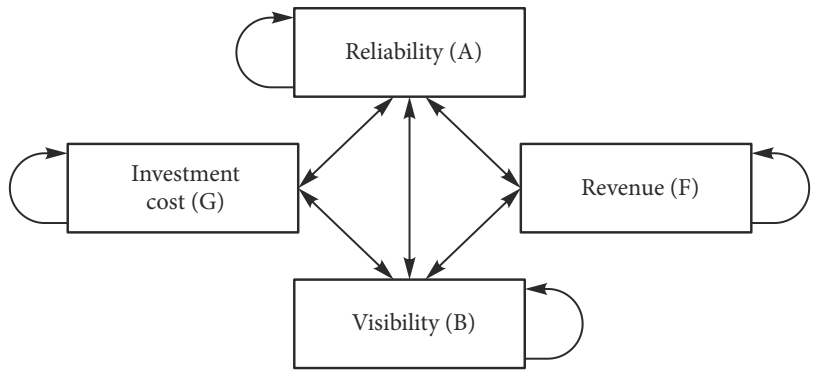

Figure 15. Relationship between dimensions preferred by rolling stock leasing company stock maintenance (Figure 15). Derived value from predictive maintenance is expected to improve reliability and reduce operational costs. Increased market share and revenue per train has an indirect financial impact on leasing companies. Dimension that is also important, and that can hinder their willingness for participation in value proposition is investment cost, which is mostly based on investment in IT systems integration. Mental shift initiatives toward eliminating their doubts related to non-realizing the added value from the innovation should also be considered.

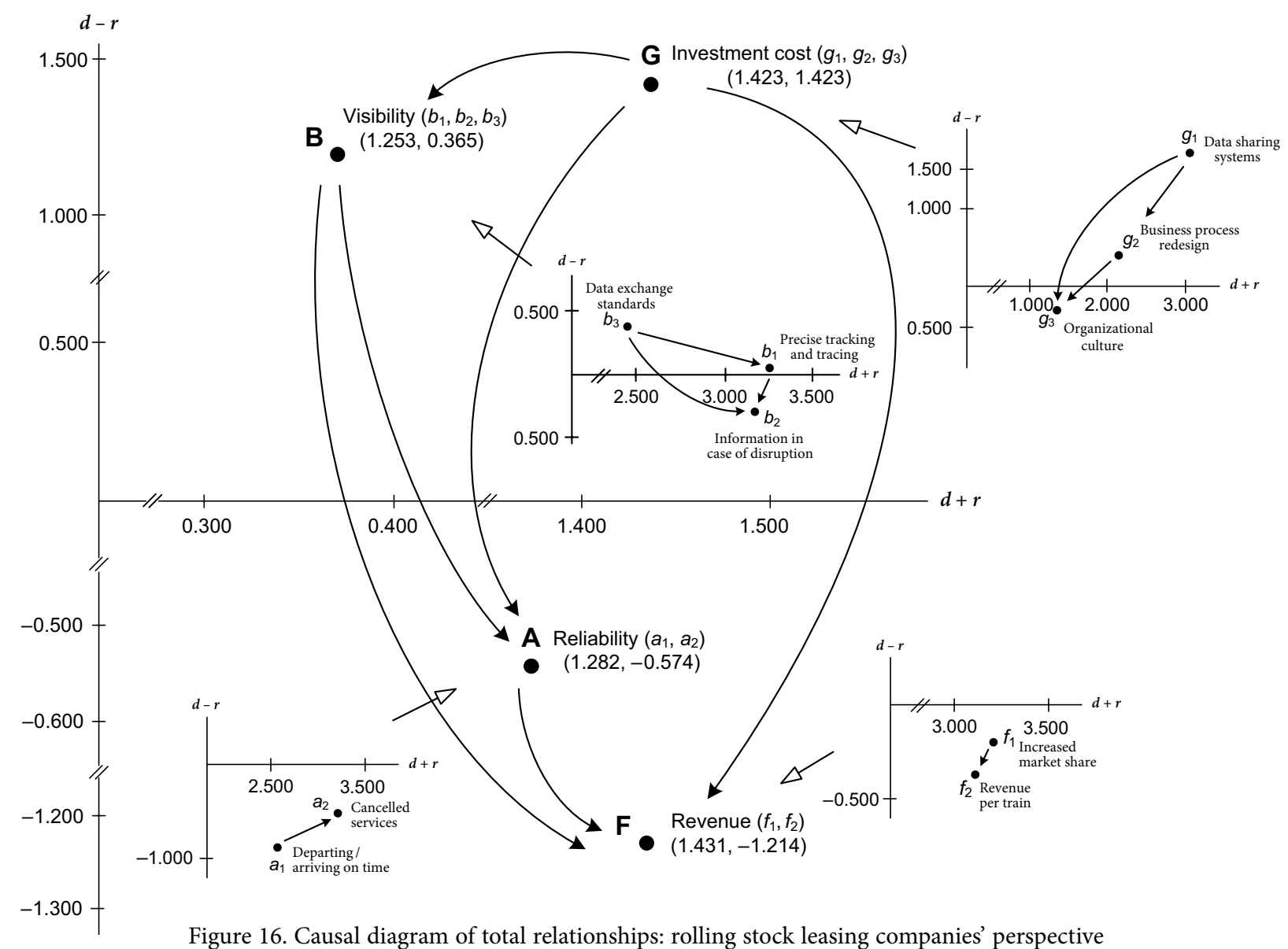
Table 10. Influential weights of DEMATEL-ANP for each criterion obtained by $\lim _{n \rightarrow \infty}\left(W^{\alpha}\right)^{n}:$ rolling stock
leasing companies' perspective

\begin{tabular}{|c|c|c|c|c|}
\hline Dimension & $\begin{array}{l}\text { Relative weight } \\
\text { of dimension }\end{array}$ & Criteria & $\begin{array}{l}\text { Relative weight } \\
\text { of criteria }\end{array}$ & Rank \\
\hline \multirow{2}{*}{ Reliability (A) } & \multirow{2}{*}{0.300} & departing / arriving on-time or within defined tolerance $a_{1}$ & 0.145 & 4 \\
\hline & & cancelled services $a_{2}$ & 0.155 & 2 \\
\hline \multirow{3}{*}{ Visibility (B) } & \multirow{3}{*}{0.243} & availability of precise tracking and tracing $b_{1}$ & 0.090 & 6 \\
\hline & & availability of information in case of disruptions $b_{2}$ & 0.094 & 5 \\
\hline & & data exchange standards supported by the ICT systems $b_{3}$ & 0.059 & 8 \\
\hline \multirow{2}{*}{ Revenue (F) } & \multirow{2}{*}{0.309} & increased market share $f_{1}$ & 0.156 & 1 \\
\hline & & revenue per $\operatorname{train} f_{2}$ & 0.153 & 3 \\
\hline \multirow{3}{*}{$\begin{array}{l}\text { Investment } \\
\text { cost }(G)\end{array}$} & \multirow{3}{*}{0.144} & data sharing systems $g_{1}$ & 0.040 & 10 \\
\hline & & business processes redesign $g_{2}$ & 0.059 & 7 \\
\hline & & organisational culture (mental shift) $g_{3}$ & 0.045 & 9 \\
\hline
\end{tabular}


The most important dimension for rolling stock leasing companies is revenue $(\mathrm{F})$ (Figure 16$)$. The revenue $(\mathrm{F})$ is influenced by investment cost $(\mathrm{G})$, reliability $(\mathrm{A})$ and visibility (B). This means that the improvement of revenue (F) depends on the improvement in investment cost $(G)$, reliability (A) and visibility (B). On the other side, in order to improve this dimension, it is needed to improve the most important performance aspects. In case of investment cost $(G)$ dimension, data sharing systems represents the most important criteria for leasing companies, and it impacts on the level of business process redesign and the need for organizational culture. Within reliability (A) dimension departing/arriving on-time would contribute to decrease of train service cancellations. This will contribute to efficiency of rolling stock leasing companies. Precise tracking and tracing $b_{1}$ represents the most important criteria in visibility (B) dimension. $b_{1}$ is supported by data exchange standards $b_{3}$ whereas both of them, $b_{1}$ and $b_{3}$, impact on availability of information in case of disruptions $b_{1}$. Precise tracking and tracing enables more efficient monitoring of assets for rolling stock leasing companies.

Table 10 presents relative weights of preferred dimensions / criteria identified by rolling stock leasing companies. Observing the relative weights of dimensions in Table 10 , it can be seen that the most important dimension is revenue $(\mathrm{F})$ with a relative weight of 0.309 . Therefore, from the aspect of rolling stock leasing companies, revenue $(F)$ is seen as the most important aspect of new value proposition, followed by reliability (A) (0.300), visibility (B) (0.243) and investment cost (G) (0.143). Table 10 also shows that the most important criteria for acceptance of new value proposition from rolling stock leasing companies are: increased market share $f_{1}(0.156)$, cancelled services $a_{2}(0.155)$ and revenue per $\operatorname{train} f_{2}(0.153)$. The least important performance aspects are organizational culture $g_{3}(0.045)$ and data sharing systems $g_{1}(0.040)$.

\subsection{Final ranking of criteria}

In previous sections, the effects of innovative value proposition are assessed from the aspect of each stakeholder involved. However, each of the involved stakeholders has different role, interest and value added to a specific value proposition. Therefore, in order to determine the absolute priorities, it is needed to rank stakeholders according to their impact or the value they deliver to a new concept. Ranking of stakeholders is conducted based on pairwise comparisons of stakeholders with respect to the innovative value proposition in rail intermodal transport chain. In total ten responses (completed pairwise comparison matrices) from University professors, researchers and consultants were collected. Respondents used Satty's 1...9 scale to express their opinion about the influence of each stakeholder in value proposition. In order to aggregate individual expert preferences a group consensus geometric mean approach was applied (Ossadnik et al. 2016). Weights of stakeholders are given in Table 11.

Now it is possible to determine the absolute utilities of all effects or attributes, which are expected by the stakeholders. These utilities are calculated by multiplying weights of stakeholders with corresponding relative weights. Some dimensions or values have a collective effect since they are identified by majority of stakeholders: Reliability (A), visibility (B) and investment cost (G) identified by five or all six stakeholders. Some other values do not have collective effect since they are identified by two stakeholders: lead time (D), efficiency of service provision (E) and flexibility (C).

The final rank of attributes identified with the new value proposition for the rail freight transport is presented on Figure 17. Based on the results, attributes that belong to reliability (A) dimension are the most important: cancelled services $a_{2}$ with weight of 0.118 , followed by departing / arriving on-time $a_{1}$ with a weight of 0.111 . Idle time $d_{2}$ is the third most important attribute $(0.102)$. Organizational

Table 11. Influential weights of stakeholders with respect to innovative value proposition in rail intermodal transport chain

\begin{tabular}{|l|c|}
\hline \multicolumn{1}{|c|}{ Stakeholder } & Weight \\
\hline Shipper & 0.328 \\
\hline LSP & 0.226 \\
\hline Railway undertaking / railway operator & 0.188 \\
\hline Terminal operator & 0.082 \\
\hline Infrastructure manager & 0.119 \\
\hline Rolling stock leasing company & 0.056 \\
\hline
\end{tabular}

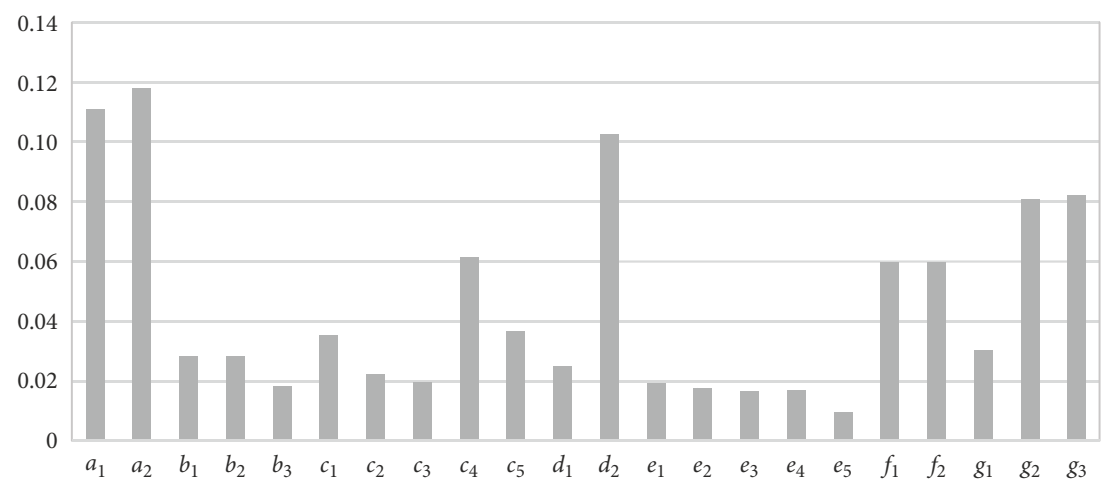

Figure 17. Absolute utilities of all effects or attributes expected by the stakeholders 
culture $g_{3}$ and business process redesign $g_{2}$ are fourth and fifth attribute with weights 0.080 and 0.082 , respectively. Ability to rebook $c_{4}$ is sixth criteria with weight of 0.061 whereas Revenue $(\mathrm{F})$ related attributes: revenue per train $f_{2}$ and increased market share $f_{1}$ follow with equivalent weights of 0.059 . Ninth and ten criteria belong to flexibility (C) dimension: time for rebooking $c_{5}$ and number of departures $c_{1}$ with weights 0.037 and 0.035 , respectively. Data sharing systems $g_{1}$ is ranked as eleventh criteria with a weight of 0.030 . Then, precise tracking and tracing $b_{1}$ and information in case of disruption $b_{2}$ follow with equivalent weights of 0.028 . Criteria ranked from fourteen to the last are as follows: time for load / unload $d_{1}(0.024)$, destinations served $c_{2}(0.022)$, utilization of train capacities $e_{1}(0.019)$, data exchange standards $b_{3}(0.018)$, empty equipment runs $e_{2}$ (0.017), productivity of terminals $e_{4}$ (0.016), total transit costs $e_{3}(0.016)$ and volume of data exchange $e_{5}(0.009)$.

\section{Concluding remarks}

Railway freight sector represents an integral part of the supply chain. Therefore, the focus of railways must be not only on improving their internal efficiency and performances but also on efficiency and performances of their respective supply chains. In that sense, railways must become an active part of a cluster of organizations, which jointly work on delivering an added value to the final customers. One of the critical facilitators of more intensive supply chain orientation of railway sector is improved coordination between rail and non-rail-related stakeholders in the transport chain.

Currently, there are difficulties for enabling the full visibility of shipments during their travelling along the railway legs due to a non-availability of the data for non-railrelated stakeholders. This has a critical impact on flexibility, reliability, lead time and costs of the service and results in lower competitiveness of rail sector. This gap between user' expectations and rail service offer can be eliminated by implementing a rail-enabled information sharing platform for improved coordination between all actors in the transport chain. In order to be successful this innovation requires alignment of interests of all stakeholders directly involved in innovation.

Therefore, the aim of this paper was to identify the attributes / dimensions that different stakeholders identify with this innovation, and through an assessment of their influences and interrelationships to evaluate the relative weights of dimensions / attributes. For this purpose, DEMATEL-ANP approach is proposed as a tool for evaluation of mutual influences and prioritizing those attributes of new value proposition, which are of highest relevance for each of the stakeholders directly involved in rail intermodal transport chain. Since different stakeholders in the transport chain have different power for initiating this innovation, the final list of attributes has been derived taking into account the importance of every stakeholder.
The approach proposed in this paper as well as the results have a number of practical implications:

"» a comprehensive and systematic approach for assessment of critical attributes for successful implementation of multi-value multi-stakeholder innovations is proposed;

"») the issue of insufficient coordination in rail intermodal transport chain is tackled from a multistakeholder perspective, the main dimensions and attributes are identified and prioritized. Obtained outputs may serve as a background and facilitator toward the implementation of this particular innovation as well as for similar innovations in rail intermodal transport. The main factors, their interrelationships and their priority may help decision makers to improve the efficiency and effectiveness of innovation implementation. Following this approach and focusing on the attributes according to their importance guarantees establishment of a collectively accepted solution and reduces the risk of failed implementation of the innovation.

"1) since currently there is a gap between expectations of users and rail freight service offer, analysis conducted in this paper has also produced a set of the most important enablers of improved competitiveness of rail freight service.

One limitation of this approach is the presence of impreciseness and vagueness in human judgments. In order to address this limitation, future research will be dedicated to incorporating vagueness through fuzzy and rough set theory. The second limitation concerns inclusion of only directly related stakeholders. Future research will also consider indirect stakeholders such as policy makers, port authorities and others. Finally, the work will be dedicated to extending the proposed DEMATEL-ANP approach and developing a model that combines DEMATEL with ANP and VIKOR for ranking or improving the priorities of attributes.

\section{Appendix. Integrated DEMATEL-ANP approach for evaluation of innovative value proposition for rail freight transport}

Following set of steps describes the methodology applied in this paper for selection of the most important attributes, which can be used to justify the implementation of information sharing platform in rail intermodal transport chain.

\section{Step 1}

Conduct the interviews with experts directly and indirectly involved in transport and logistics and design the questionnaires based on experts' knowledge, experience and literature survey.

\section{Step 2}

Gather experts' opinion about the attributes and select the preferred subset based on TFNs. 


\section{Step 3}

Synthesize the experts' opinions regarding the relative importance of stakeholders in transport chain and determine their relative weight in the rail intermodal transport chain.

From the perspective of each type of stakeholder $s=1$, $\ldots, S$ in the value network (shippers, LSPs, terminal operators, railway undertakings / railway operators, infrastructure managers, rolling stock leasing companies) perform the following set of steps:

DEMATEL methodology to determine interdependence among dimensions and attributes (Step 4 - Step 7).

\section{Step 4}

Calculate the average direct relation matrix $A$.

Experts are asked to compare the dimensions and attributes pairwise in terms of influence and direction. This is done based on comparison scale 0-4 composed from five levels: no influence (0), low influence (1), medium influence (2), high influence (3) and very high influence (4). Based on these inputs a matrix $A$ of $n \times n$ dimensions, known as the average direct relation matrix is constructed - Equation (A1). Element $a_{i j}$ represents the degree to which the dimension / attribute $i$ affects the dimension / attribute $j$ and it is computed as an average of inputs of all experts of type $s$ for a given $i-j$ pair of dimensions / attributes.

$$
A=\left[\begin{array}{ccccc}
a_{11} & \ldots & a_{1 j} & \ldots & a_{1 n} \\
\vdots & & \vdots & & \vdots \\
a_{i 1} & & a_{i j} & & a_{i n} \\
\vdots & & \vdots & & \vdots \\
a_{n 1} & & a_{n j} & & a_{n n}
\end{array}\right] .
$$

\section{Step 5}

Normalization of the direct relation matrix.

Matrix $N$ as the normalized version of the average direct relation matrix represents a multiplication of matrix $A$ and $k$ :

$$
N=A \cdot k
$$

where:

$$
k=\min \left(\frac{1}{\max _{i} \sum_{j=1}^{n}\left|a_{i j}\right|}, \frac{1}{\max _{j} \sum_{j=1}^{n}\left|a_{i j}\right|}\right) .
$$

\section{Step 6}

Determine the total relation matrix.

Based on the estimated direct influence of the dimensions / attributes the total influence matrix $T=\left[t_{i j}\right]_{n \times n}$ can be obtained from matrix $N$ by applying the transition theory and summing up all direct and indirect effects:

$$
T=N+N+N+\cdots+N^{m}=N \cdot(I-N)^{-1},
$$

when $m \rightarrow \infty$,

where: $I$ is an $n \times n$ identity matrix.

\section{Step 7}

Obtaining the NRM.
In order to draw the NRM it is needed to calculate the sums of rows $r$ and columns $c$ of the total relation matrix $T$ :

$$
\begin{aligned}
& r=\left(r_{i}\right)_{n \times 1}=\left[\sum_{j=1}^{n} t_{i j}\right]_{n \times 1} ; \\
& c=\left(c_{j}\right)_{n \times 1}=\left(c_{j}\right)_{1 \times n}^{\prime}=\left[\sum_{i=1}^{n} t_{i j}\right]_{1 \times n}^{\prime} .
\end{aligned}
$$

The value $r_{i}$ represents the total effect, both direct and indirect, that dimension / attribute $i$ has on the other dimensions / attributes. The value $c_{j}$ as the sum of the $j$ th column in matrix $T$ represents the total effects, both direct and indirect, that dimension / attribute $j$ has received from other dimensions / attributes $j$. The influence matrix $T$ can be divided into $T_{D}$ based on dimensions and $T_{A}$ based on attributes.

$$
\begin{aligned}
& \begin{array}{ccccc}
D_{1} & \ldots & D_{i} & \ldots & D_{n} \\
a_{11} \ldots a_{1 m 1} & \ldots & a_{i 1} \ldots a_{i m_{i}} & \ldots & a_{n_{1}} \ldots a_{n m_{n}}
\end{array} \\
& \begin{array}{c}
a_{11} \\
D_{1} \vdots \\
a_{1 m_{1}} \\
a_{i 1} \\
D_{i} \\
a_{i m_{i}} \\
D_{n 1} \\
D_{n} \vdots \\
a_{n m_{n}}
\end{array} \quad\left[\begin{array}{ccccc}
T_{a}^{11} & \cdots & T_{a}^{1 j} & \cdots & T_{a}^{1 n} \\
\vdots & & \vdots & & \vdots \\
T_{a}^{i 1} & \cdots & T_{a}^{i j} & \cdots & T_{a}^{i n} \\
\vdots & & \vdots & \ddots & \vdots \\
T_{a}^{n 1} & \cdots & T_{a}^{n j} & \cdots & T_{a}^{n n}
\end{array}\right] .
\end{aligned}
$$

Matrix $T_{a}^{11}$ represents a matrix of attributes that belong to the group $D_{1}$ as well as the influences in respect of the attributes from the dimension $D_{1}$ - Equation (8), whereas $T_{a}^{12}$ is a matrix of $D_{2}$ related attributes and influences in respect of the attributes from the dimension $D_{2}$ and so on.

$$
T_{a}^{11}=\left[\begin{array}{ccccc}
t_{a^{11}}^{11} & \cdots & t_{a^{1 j}}^{11} & \cdots & t_{a^{1 m_{1}}}^{11} \\
\vdots & & \vdots & & \vdots \\
t^{11} & \cdots & t_{a^{i j}}^{11} & \cdots & t^{11} \\
\vdots & & \vdots & \ddots & \vdots \\
t^{i m} \\
a^{11} & \cdots & t^{11} & \cdots & t^{11} \\
a^{m 1 j} & & & a^{m 1 m 1}
\end{array}\right] .
$$

NRM represents a diagram, which illustrates the roles that the dimensions / attributes have in the evaluation of the innovative value proposition with horizontal axis $(r+$ $c)$ and the vertical axis $(r-c)$ (Dalvi-Esfahani et al. 2019). The sum $\left(r_{i}+c_{j}\right)$, also called "prominence", represents the importance that dimension / attribute $i$ plays in the system, whereas the difference $\left(r_{i}-c_{j}\right)$, known as "relation", shows the net effect that the dimension / attribute $i$ contributes to the evaluation of the innovative value proposition. Positive result of $\left(r_{i}-c_{j}\right)$ means that the factor $i$ is a net causer, whereas when $\left(r_{i}-c_{j}\right)$ is negative the factor $i$ is a net receiver. In order to obtain an appropriate diagram, it is needed to set the influence level threshold value.

ANP methodology to determine relative weights (Steps 8-11).

Relative weights of attributes will be calculated based on combination of ANP and DEMATEL. This enables overcoming of some deficiencies of traditional ANP 
method. More precisely, in case of DEMATEL method, the levels of interdependences of attributes do not have reciprocal values like in traditional ANP approach (Yang, Tzeng 2011). Therefore, to calculate the relative weight of attributes the total relation matrix $T$ will be used in order to avoid the shortcomings of traditional ANP approach (Vujanović et al. 2012). Following four steps compose the essence of DEMATEL and ANP combination.

\section{Step 8}

Pairwise comparison of dimensions / attributes in order to form the unweighted supermatrix.

Supermatrix in general form looks like follows (Supeekit et al. 2016):

$$
\begin{aligned}
& \begin{array}{ccccc}
D_{1} & \ldots & D_{i} & \ldots & D_{n} \\
a_{11} \ldots, a_{1 m 1} & \ldots & a_{i 1} \ldots a_{i m_{i}} & \ldots & a_{n_{1}} \ldots a_{n m_{n}}
\end{array}
\end{aligned}
$$

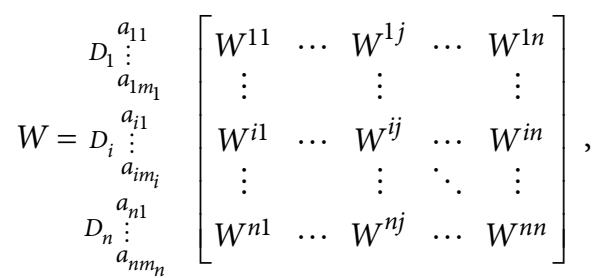

where: $D_{n}$ represents the $n$th dimension (cluster), $a_{n m}$ denotes the $n$th atribute in $m$ th dimension and $W_{i j}$ is the principal eigenvector of the impact of the attributes belonging to the $j$ th dimension compared to the $i$ th dimension (cluster). In case if the $j$ th dimension has no influence then $W_{i j}=[0]$.

Normalization of total influence matrix $T_{a}$ represents necessary precondition for obtaining unweighted super matrix. Normalized total influence matrix $T_{a}^{\alpha}$ looks like follows:

$$
\begin{aligned}
& \begin{array}{ccccc}
D_{1} & \ldots & D_{i} & \ldots & D_{n} \\
a_{11} \ldots a_{1 m 1} & \ldots & a_{i 1} \ldots a_{i m_{i}} & \ldots & a_{n_{1}} \ldots a_{n m_{n}}
\end{array}
\end{aligned}
$$

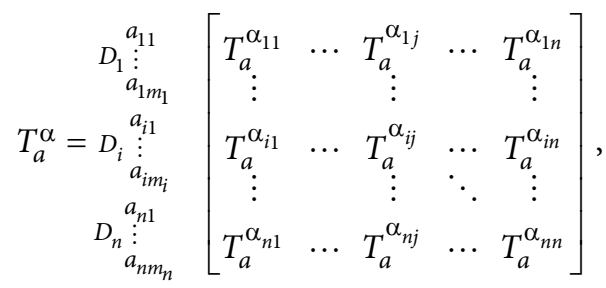

where: $T_{a}^{\alpha_{11}}$ represents a normalized sum of factor influences $a_{11}, \ldots, a_{1 m 1}$ related to attributes, which belong to dimension $D_{1}$ and calculated as follows:

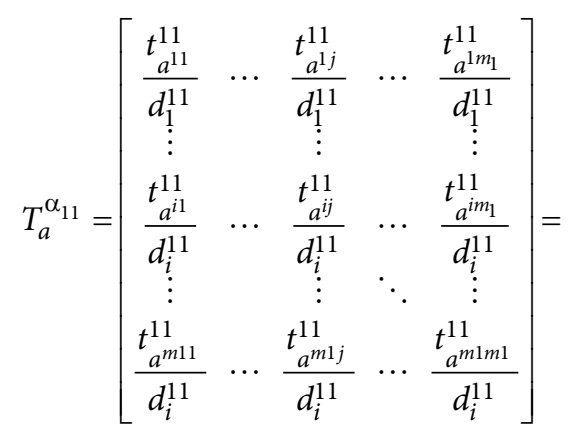

$$
\left[\begin{array}{ccccc}
t_{a_{11}^{\alpha_{11}}}^{\alpha_{1}} & \cdots & t_{a^{1 j}}^{\alpha_{11}} & \cdots & t_{a^{11}}^{\alpha_{11}} \\
\vdots & & \vdots & & \vdots \\
t_{a_{11}}^{\alpha_{11}} & \cdots & t_{a_{11}}^{\alpha_{11}} & \cdots & t_{a_{11}}^{\alpha_{11}} \\
\vdots & & \vdots & \ddots & \vdots \\
t_{a^{m 11}}^{\alpha_{11}} & \cdots & t_{a^{m 1 j}}^{\alpha_{11}} & \cdots & t_{a^{m 1 m 1}}^{\alpha_{11}}
\end{array}\right],
$$

where: $d_{i}^{11}$ represents sum of influences of factors $a_{11}, \ldots$, $a_{1 m 1}$ related to the first dimension $D_{1}$ :

$$
d_{i}^{11}=\sum_{j=1}^{m_{1}} t_{a^{1 j}}^{11} \text { for } i=1,2, \ldots, m_{1} .
$$

Elements $t_{{ }^{11}}^{11}$ represent values of attribute impacts $a_{11}$, ..., $a_{1 m 1}$ in relation to attributes that belong to the dimension $D_{1}$, whereas elements $t_{a_{11}}^{\alpha_{11}}$ are their normalized values.

Unweighted matrix $W-$ Equation (9) - is composed from normalized values of attribute influences $T_{c}^{\alpha_{n n}}$, which are all computed following the above explained procedure. Component matrices within supermatrix $W$ represent the values of attribute influences between different dimensions. For example, the matrix $W^{11}$ represents the values of attribute impacts from the dimension $D_{1}$ in relation to attributes from $D_{1}$ dimension according to:

$$
\begin{aligned}
& \begin{array}{llllll}
c_{11} & \cdots & & c_{1 j} & \cdots & c_{1 m 1}
\end{array}
\end{aligned}
$$

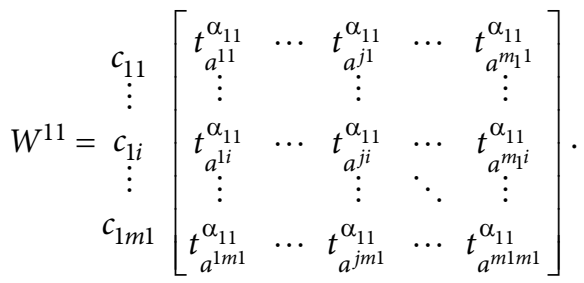

\section{Step 9}

Calculation of the weighted supermatrix. Weighted supermatrix $W_{W}$ is obtained based on the same procedure, using in this case the normalized total influence matrix $T_{D}$ and unweighted supermatrix:

$$
T_{D}=\left[\begin{array}{ccccc}
t_{D}^{11} & \cdots & t_{D}^{1 j} & \cdots & t_{D}^{1 n} \\
\vdots & & \vdots & & \vdots \\
t_{D}^{i 1} & & t_{D}^{i j} & & t_{D}^{i n} \\
\vdots & & \vdots & & \vdots \\
t_{D}^{n 1} & \cdots & t_{D}^{n j} & \cdots & t_{D}^{n n}
\end{array}\right],
$$

where: $t_{D}^{i j}$ represents the sum of influences from the ma$\operatorname{trix} T_{a}^{i j}$.

Normalization is performed as follows:

$$
T_{D}^{\alpha}=\left[\begin{array}{ccccc}
\frac{t_{D}^{11}}{d_{1}} & \cdots & \frac{t_{D}^{1 j}}{d_{1}} & \cdots & \frac{t_{D}^{1 n}}{d_{1}} \\
\vdots & & \vdots & & \vdots \\
\frac{t_{D}^{i 1}}{d_{i}} & \cdots & \frac{t_{D}^{i j}}{d_{i}} & \cdots & \frac{t_{D}^{i n}}{d_{i}} \\
\vdots & & \vdots & & \vdots \\
\frac{t_{D}^{n 1}}{d_{n}} & \cdots & \frac{t_{D}^{n j}}{d_{n}} & \cdots & \frac{t_{D}^{n n}}{d_{n}}
\end{array}\right]=
$$




$$
\left[\begin{array}{ccccc}
t_{D}^{\alpha_{11}} & \cdots & t_{D}^{\alpha_{1 j}} & \cdots & t_{D}^{\alpha_{1 n}} \\
\vdots & & \vdots & & \vdots \\
t_{D}^{\alpha_{i 1}} & \cdots & t_{D}^{\alpha_{i j}} & \cdots & t_{D}^{\alpha_{i n}} \\
\vdots & & \vdots & \ddots & \vdots \\
t_{D}^{\alpha_{n 1}} & \cdots & t_{D}^{\alpha_{n j}} & \cdots & t_{D}^{\alpha_{n n}}
\end{array}\right]
$$

where:

$$
d_{i}=\sum_{j=1}^{n} t_{d}^{i j}, \text { for } i=1,2, \ldots, n .
$$

Weighted supermatrix $W_{W}$ is based on the integration of unweighted matrix $W$ into the normalized matrix of attribute influences $T_{D}^{\alpha}$ according to:

$$
W_{w}=\left[\begin{array}{ccccc}
t_{D}^{\alpha_{11}} \cdot W^{11} & t_{D}^{\alpha_{21}} \cdot W^{12} & \cdots & \cdots & t_{D}^{\alpha_{n 1}} \cdot W^{1 n} \\
t_{D}^{\alpha_{12}} \cdot W^{21} & t_{D}^{\alpha_{22}} \cdot W^{22} & \vdots & & \vdots \\
\vdots & \cdots & t_{D}^{\alpha_{i j}} \cdot W^{i j} & \cdots & t_{D}^{\alpha_{n j}} \cdot W^{i n} \\
\vdots & & \vdots & \ddots & \vdots \\
t_{D}^{\alpha_{1 n}} \cdot W^{n 1} & t_{D}^{\alpha_{2 n}} \cdot W^{n 2} & \cdots & \cdots & t_{D}^{\alpha_{n n}} \cdot W^{n n}
\end{array}\right] .
$$

\section{Step 10}

Calculation of limited supermatrix. Limited supermatrix is calculated by raising the weighted supermatrix $W_{W}$ to a sufficient power $k$, until the supermatrix has converged and become a long term stable supermatrix.

Vectors of the limited supermatrix represent the relative weights of each attribute in respect to the evaluation of the innovative value proposition of rail intermodal freight transport:

$$
\lim _{k \rightarrow \infty} W_{w}^{k}
$$

\section{Step 11}

Determining the final weights of attributes.

The final weights of attributes are obtained by multiplying the weights obtained in Step 10 by the relative priority of each stakeholder in the value network.

\section{References}

Bongo, M. F.; Alimpangog, K. M. S.; Loar, J. F.; Montefalcon, J. A.; Ocampo, L. A. 2018. An application of DEMATEL-ANP and PROMETHEE II approach for air traffic controllers' workload stress problem: a case of Mactan civil aviation authority of the Philippines, Journal of Air Transport Management 68: 198-213. https://doi.org/10.1016/j.jairtraman.2017.10.001

Bongo, M. F; Ocampo, L. A. 2017. A hybrid fuzzy MCDM approach for mitigating airport congestion: a case in Ninoy Aquino international airport, Journal of Air Transport Management 63: 1-16.

https://doi.org/10.1016/j.jairtraman.2017.05.004

Brooks, M. R.; Puckett, S. M.; Hensher, D. A.; Sammons, A. 2012. Understanding mode choice decisions: a study of Australian freight shippers, Maritime Economics \& Logistics 14(3): 274299. https://doi.org/10.1057/mel.2012.8

Büyüközkan, G.; Güleryüz, S. 2016. An integrated DEMATEL-
ANP approach for renewable energy resources selection in Turkey, International Journal of Production Economics 182: 435-448. https://doi.org/10.1016/j.ijpe.2016.09.015

Chen, F.-H.; Hsu, T.-S.; Tzeng, G.-H. 2011. A balanced scorecard approach to establish a performance evaluation and relationship model for hot spring hotels based on a hybrid MCDM model combining DEMATEL and ANP, International Journal of Hospitality Management 30(4): 908-932. https://doi.org/10.1016/j.ijhm.2011.02.001

Chen, I.-S. 2016. A combined MCDM model based on DEMATEL and ANP for the selection of airline service quality improvement criteria: a study based on the Taiwanese airline industry, Journal of Air Transport Management 57: 7-18. https://doi.org/10.1016/j.jairtraman.2016.07.004

Clusters 2.0. 2019. D.2.5: Handbook for Smart Clusters Development. Clusters 2.0 - Open Network of Hyper Connected Logistics Clusters Towards Physical Internet. European Union's Horizon 2020 Research and Innovation Programme under Grant Agreement No 723265. 57 p. Available from Internet: http://www.clusters20.eu/wp-content/uploads/2019/09/Clusters_2.0_D2.5-Handbook-for-Smart-Clusters-DevelopmentFINAL-VERSION_IBI-20190829-1.pdf

Combes, F. 2012. Empirical evaluation of economic order quantity model for choice of shipment size in freight transport, Transportation Research Record: Journal of the Transportation Research Board 2269: 92-98. https://doi.org/10.3141/2269-11

Dalvi-Esfahani, M.; Niknafs, A.; Kuss, D. J.; Nilashi, M.; Afrough, S. 2019. Social media addiction: applying the DEMATEL approach, Telematics and Informatics 43: 101250.

https://doi.org/10.1016/j.tele.2019.101250

Danielis, R.; Marcucci, E.; Rotaris, L. 2005. Logistics managers' stated preferences for freight service attributes, Transportation Research Part E: Logistics and Transportation Review 41(3): 201-215. https://doi.org/10.1016/j.tre.2004.04.003

Dehdasht, G.; Zin, R. M.; Ferwati, M. S.; Abdullahi, M. M.; Keyvanfar, A.; McCaffer, R. 2017. DEMATEL-ANP risk assessment in oil and gas construction projects, Sustainability 9(8): 1420. https://doi.org/10.3390/su9081420

Dinçer, H.; Hacıoğlu, Ü.; Yüksel, S. 2017. Balanced scorecard based performance measurement of European airlines using a hybrid multicriteria decision making approach under the fuzzy environment, Journal of Air Transport Management 63: 17-33. https://doi.org/10.1016/j.jairtraman.2017.05.005

Fontela, E.; Gabus, A. 1974. DEMATEL: Innovative Methods. Science and Human Affairs Program of the Battelle Memorial Institute of Geneva, Geneva, Switzerland.

Fontela, E.; Gabus, A. 1976. The DEMATEL Observer. Science and Human Affairs Program of the Battelle Memorial Institute of Geneva, Geneva, Switzerland.

García-Menéndez, L.; Martínez-Zarzoso, I.; Perez-Garcia, E. M. 2006. Transport Logistics and Modal Split of Spanish Exports to Europe: Empirical Evidence. Social Science Research Network (SSRN). 26 p. https://doi.org/10.2139/ssrn.903048

Gleave, S. D.; Dionori, F.; Casullo, L.; Ellis, S.; Ranghetti, D.; Bablinski, K.; Vollath, C.; Soutra, C. 2015. Freight on Road: Why EU Shippers Prefer Truck to Train. European Parliament's Committee on Transport and Tourism, European Union. 78 p. Available from Internet: https://www.europarl.europa.eu/RegData/etudes/STUD/2015/540338/IPOL_STU(2015)540338_ EN.pdf

Gölcük, İ.; Baykasoğlu, A. 2016. An analysis of DEMATEL approaches for criteria interaction handling within ANP, Expert Systems with Applications 46: 346-366. https://doi.org/10.1016/j.eswa.2015.10.041 
Gudiel Pineda, P. J.; Liou, J. J. H.; Hsu, C.-C.; Chuang, Y.-C. 2018. An integrated MCDM model for improving airline operational and financial performance, Journal of Air Transport Management 68: 103-117.

https://doi.org/10.1016/j.jairtraman.2017.06.003

Ha, M.-H.; Yang, Z. 2017. Comparative analysis of port performance indicators: independency and interdependency, Transportation Research Part A: Policy and Practice 103: 264-278. https://doi.org/10.1016/j.tra.2017.06.013

Ha, M.-H.; Yang, Z.; Notteboom, T.; Ng, A. K. Y.; Heo, M.-W. 2017. Revisiting port performance measurement: a hybrid multi-stakeholder framework for the modelling of port performance indicators, Transportation Research Part E: Logistics and Transportation Review 103: 1-16.

https://doi.org/10.1016/j.tre.2017.04.008

Hsu, C.-C.; Liou, J. J. H. 2013. An outsourcing provider decision model for the airline industry, Journal of Air Transport Management 28: 40-46.

https://doi.org/10.1016/j.jairtraman.2012.12.009

Hsu, C.-C.; Liou, J. J. H.; Lo, H.-W.; Wang, Y.-C. 2018. Using a hybrid method for evaluating and improving the service quality of public bike-sharing systems, Journal of Cleaner Production 202: 1131-1144. https://doi.org/10.1016/j.jclepro.2018.08.193

Ishfaq, R. 2012. Resilience through flexibility in transportation operations, International Journal of Logistics Research and Applications: a Leading Journal of Supply Chain Management 15(4): 215-229. https://doi.org/10.1080/13675567.2012.709835

Kaplinsky, R.; Morris, M. 2001. A Handbook for Value Chain Research. International Development Research Centre, Ottawa, Canada. 113 p.

Karaşan, A.; Kahraman, C. 2019. A novel intuitionistic fuzzy DEMATEL-ANP-TOPSIS integrated methodology for freight village location selection, Journal of Intelligent \& Fuzzy Systems 36(2): 1335-1352. https://doi.org/10.3233/JIFS-17169

Kheybari, S.; Rezaie, F. M.; Farazmand, H. 2020. Analytic network process: an overview of applications, Applied Mathematics and Computation 367: 124780.

https://doi.org/10.1016/j.amc.2019.124780

Kijewska, K.; Torbacki, W.; Iwan, S. 2018. Application of AHP and DEMATEL methods in choosing and analysing the measures for the distribution of goods in Szczecin region, Sustainability 10(7): 2365. https://doi.org/10.3390/su10072365

Kim, H.-C.; Nicholson, A.; Kusumastuti, D. 2017. Analysing freight shippers' mode choice preference heterogeneity using latent class modelling, Transportation Research Procedia 25: 1109-1125. https://doi.org/10.1016/j.trpro.2017.05.123

Kumar, A.; Anbanandam, R. 2020. Analyzing interrelationships and prioritising the factors influencing sustainable intermodal freight transport system: a grey-DANP approach, Journal of Cleaner Production 252: 119769.

https://doi.org/10.1016/j.jclepro.2019.119769

Kundakcı, N.; Adalı, E. A.; Işık, A. T. 2014. Combination of DEMATEL and ANP for the cargo shipping company selection problem, International Journal of Engineering Management and Economics 4(2): 99-116.

https://doi.org/10.1504/IJEME.2014.066574

Lee, K.-C.; Tsai, W.-H.; Yang, C.-H.; Lin, Y.-Z. 2018. An MCDM approach for selecting green aviation fleet program management strategies under multi-resource limitations, Journal of Air Transport Management 68: 76-85.

https://doi.org/10.1016/j.jairtraman.2017.06.011

Lin, C.-L.; Hsieh, M.-S.; Tzeng, G.-H. 2010. Evaluating vehicle telematics system by using a novel MCDM techniques with dependence and feedback, Expert Systems with Applications 37(10): 6723-6736. https://doi.org/10.1016/j.eswa.2010.01.014
Liou, J. J. H.; Hsu, C.-C.; Chen, Y.-S. 2014. Improving transportation service quality based on information fusion, Transportation Research Part A: Policy and Practice 67: 225-239.

https://doi.org/10.1016/j.tra.2014.07.007

Liou, J. J. H.; Tzeng, G.-H.; Chang, H.-C. 2007. Airline safety measurement using a hybrid model, Journal of Air Transport Management 13(4): 243-249.

https://doi.org/10.1016/j.jairtraman.2007.04.008

Liu, C.-H.; Tzeng, G.-H.; Lee, M.-H.; Lee, P.-Y. 2013. Improving metro-airport connection service for tourism development: using hybrid MCDM models, Tourism Management Perspectives 6: 95-107. https://doi.org/10.1016/j.tmp.2012.09.004

Lu, M.-T.; Hsu, C.-C.; Liou, J. J. H.; Lo, H.-W. 2018. A hybrid MCDM and sustainability-balanced scorecard model to establish sustainable performance evaluation for international airports, Journal of Air Transport Management 71: 9-19. https://doi.org/10.1016/j.jairtraman.2018.05.008

Moschovou, T. P.; Giannopoulos, G. A. 2012. Modeling freight mode choice in Greece, Procedia - Social and Behavioral Sciences 48: 597-611.

https://doi.org/10.1016/j.sbspro.2012.06.1038

Ossadnik, W.; Schinke, S.; Kaspar, R. H. 2016. Group aggregation techniques for analytic hierarchy process and analytic network process: a comparative analysis, Group Decision and Negotiation 25(2): 421-457.

https://doi.org/10.1007/s10726-015-9448-4

Pamučar, D.; Ćirović, G. 2015. The selection of transport and handling resources in logistics centers using multi-attributive border approximation area comparison (MABAC), Expert Systems with Applications 42(6): 3016-3028.

https://doi.org/10.1016/j.eswa.2014.11.057

Pamučar, D.; Đorović, B.; Božanić, D.; Ćirović, G. 2012. Modification of the dynamic scale of marks in analytic hierarchy process (AHP) and analytic network approach (ANP) through application of fuzzy approach, Scientific Research and Essays 7(1): 24-37.

Patterson, Z.; Ewing, G. O.; Haider, M. 2007. Shipper preferences suggest strong mistrust of rail: results from stated preference carrier choice survey for Quebec City-Windsor Corridor in Canada, Transportation Research Record: Journal of the Transportation Research Board 2008: 67-74. https://doi. org/10.3141/2008-09

Quezada, L. E.; López-Ospina, H. A.; Palominos, P. I.; Oddershede, A. M. 2018. Identifying causal relationships in strategy maps using ANP and DEMATEL, Computers \& Industrial Engineering 118: 170-179. https://doi.org/10.1016/j.cie.2018.02.020

Ranjan, R.; Chatterjee, P.; Chakraborty, S. 2015. Evaluating performance of engineering departments in an Indian University using DEMATEL and compromise ranking methods, OPSEARCH 52(2): 307-328.

https://doi.org/10.1007/s12597-014-0186-1

Ranjan, R.; Chatterjee, P.; Chakraborty, S. 2016. Performance evaluation of Indian railway zones using DEMATEL and VIKOR methods, Benchmarking: an International Journal 23(1): 78-95. https://doi.org/10.1108/BIJ-09-2014-0088

Saaty, T. L. 2008. Decision making with the analytic hierarchy process, International Journal of Services Sciences 1(1): 83-98. https://doi.org/10.1504/IJSSCI.2008.017590

Saaty, T. L.; Vargas, L. G. 2006. Decision Making with the Analytic Network Process: Economic, Political, Social and Technological Applications with Benefits, Opportunities, Costs and Risks. Springer. 280 p. https://doi.org/10.1007/0-387-33987-6

Samimi, A.; Mohammadian, A.; Kawamura, K. 2010. A behavioral freight movement microsimulation model: method 
and data, Transportation Letters: the International Journal of Transportation Research 2(1): 53-62. https://doi.org/10.3328/TL.2010.02.01.53-62

Shaik, M. N.; Abdul-Kader, W. 2018. A hybrid multiple criteria decision making approach for measuring comprehensive performance of reverse logistics enterprises, Computers \& Industrial Engineering 123: 9-25.

https://doi.org/10.1016/j.cie.2018.06.007

Shin, S.; Roh, H.-S.; Hur, S. H. 2019. Characteristics analysis of freight mode choice model according to the introduction of a new freight transport system, Sustainability 11(4): 1209. https://doi.org/10.3390/su11041209

Smart-Rail. 2016. D7.4: Alignment of the Value Case of Involved Stakeholders. Document ID: Smart-Rail-D7.4-v1.0. SmartRail: Smart Supply Chain Oriented Rail Freight Services. European Union's Horizon 2020 Research and Innovation Programme under Grant Agreement No 636071. 52 p. Available from Internet: https://smartrail-project.eu/download/ public_reports/D7.4\%20-\%20v1.0\%20Alignment $\% 20$ of $\% 20$ the $\% 20$ value $\% 20$ case $\% 20$ of $\% 20$ involved $\% 20$ stakeholders.pdf

Solakivi, T.; Ojala, L. 2017. Determinants of carrier selection: updating the survey methodology into the 21st century, Transportation Research Procedia 25: 511-530. https://doi.org/10.1016/j.trpro.2017.05.433

Supeekit, T.; Somboonwiwat, T.; Kritchanchai, D. 2016. DEMATEL-modified ANP to evaluate internal hospital supply chain performance, Computers \& Industrial Engineering 102: 318-330. https://doi.org/10.1016/j.cie.2016.07.019

Tafreshi, P. F.; Aghdaie, M. H.; Behzadian, M.; Abadi, M. G. 2016. Developing a group decision support system for advertising media evaluation: a case in the Middle East, Group Decision and Negotiation 25(5): 1021-1048. https://doi.org/10.1007/s10726-015-9464-4

Vujanović, D.; Momčilović, V.; Bojović, N.; Papić, V. 2012. Evaluation of vehicle fleet maintenance management indicators by application of DEMATEL and ANP, Expert Systems with Applications 39(12): 10552-10563.

https://doi.org/10.1016/j.eswa.2012.02.159

Wambua, J.; Mukulu, E.; Waiganjo, E. 2017. Cost as a factor of outsourcing third-party logistics providers and the performance of food and beverages manufacturing companies in Kenya, International Journal of Academic Research in Business and Social Sciences 7(2): 343-356.

Yang, J. L.; Tzeng, G.-H. 2011. An integrated MCDM technique combined with DEMATEL for a novel cluster-weighted with ANP method, Expert Systems with Applications 38(3): 14171424. https://doi.org/10.1016/j.eswa.2010.07.048

Yazdani, M.; Chatterjee, P.; Zavadskas, E. K.; Hashemkhani Zolfani, S. 2017. Integrated QFD-MCDM framework for green supplier selection, Journal of Cleaner Production 142: 37283740. https://doi.org/10.1016/j.jclepro.2016.10.095

Yazdani, M.; Pamucar, D.; Chatterjee, P.; Chakraborty, S. 2020. Development of a decision support framework for sustainable freight transport system evaluation using rough numbers, International Journal of Production Research 58(14): 4325-4351. https://doi.org/10.1080/00207543.2019.1651945

Zhan, Q.; Zheng, W.; Zhao, B. 2017. A hybrid human and organizational analysis method for railway accidents based on HFACS-railway accidents (HFACS-RAs), Safety Science 91: 232-250. https://doi.org/10.1016/j.ssci.2016.08.017 